\title{
Blue flicker modifies the subfoveal choroidal blood flow in the human eye
}

\author{
par \\ Marcelo Alejandro Wajszilber
}

Ecole d'Optometrie

Universite de Montreal

Thèse présentée à la Faculté des études supérieures en vue de l'obtention du grade de Maîtrise (M.Sc.)

en sciences de la vision

option sciences fondamentales et appliquées

August 2007

(C) Marcelo Wajszilber, 2007

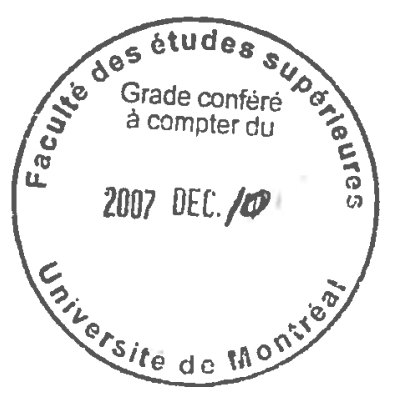


wh

3

058

2007

$\mathrm{V} \cdot 1002$ 
Direction des bibliothèques

\section{AVIS}

L'auteur a autorisé l'Université de Montréal à reproduire et diffuser, en totalité ou en partie, par quelque moyen que ce soit et sur quelque support que ce soit, et exclusivement à des fins non lucratives d'enseignement et de recherche, des copies de ce mémoire ou de cette thèse.

L'auteur et les coauteurs le cas échéant conservent la propriété du droit d'auteur et des droits moraux qui protègent ce document. Ni la thèse ou le mémoire, ni des extraits substantiels de ce document, ne doivent être imprimés ou autrement reproduits sans l'autorisation de l'auteur.

Afin de se conformer à la Loi canadienne sur la protection des renseignements personnels, quelques formulaires secondaires, coordonnées ou signatures intégrées au texte ont pu ètre enlevés de ce document. Bien que cela ait pu affecter la pagination, il n'y a aucun contenu manquant.

\section{NOTICE}

The author of this thesis or dissertation has granted a nonexclusive license allowing Universite de Montreal to reproduce and publish the document, in part or in whole, and in any format, solely for noncommercial educational and research purposes.

The author and co-authors if applicable retain copyright ownership and moral rights in this document. Neither the whole thesis or dissertation, nor substantial extracts from it, may be printed or otherwise reproduced without the author's permission.

In compliance with the Canadian Privacy Act some supporting forms, contact information or signatures may have been removed from the document. While this may affect the document page count, it does not represent any loss of content from the document. 
Université de Montréal

Faculté des études supérieures

Cette thèse intitulée :

\title{
Blue flicker modifies the subfoveal choroidal blood flow in the human eye
}

présentée par :

Marcelo Wajszilber

a été évaluée par un jury composé des personnes suivantes :

\author{
Prof. Dr.John Lovasik OD,PhD, \\ Directeur de recherche \\ Prof .Dr.Helene Kergoat OD,PhD, \\ Membre du jury
}

Prof. Dr Etty Bitton OD,MSc, Membre du jury 


\section{Résumé}

L'objectif de cette étude était de démontrer le lien entre le débit sanguin choroïdien $(\mathrm{ChBF})$ et l'activité des photorécepteurs induite par la lumière. Dans le passé, cet effet a déjà été démontré pour la circulation rétinienne mais a été réfuté pour la circulation choroïdienne. Dix sujets en bonne santé ont participé à cette étude.

Le débit sanguin a été mesuré à l'aide d'un laser Doppler infrarouge pendant la stimulation avec une lumière bleue clignotante. Les photorécepteurs rétiniens ont été stimulés par 24 fréquences de clignotement comprises entre 1 et $64 \mathrm{~Hz}$. Pour chaque fréquence, nous avons augmenté l'intensité lumineuse de 1 unité logarithmique à 4 unités logarithmique. Ensuite, nous avons diminué l'intensité de 1 unité logarithmique par étape pour retourner au point de départ, c'est-à-dire de $375 \mathrm{~cd} / \mathrm{m}$ à $0,00375 \mathrm{~cd} / \mathrm{m}$. En même temps, nous avons mesuré l'activité électrique rétinienne avec l'électrorétinogramme par flash (ERG's) pour déterminer le degré d'activité des cônes bleus (S) et des bâtonnets avec la stimulation bleue. Les mesures du ChBF normalisées et moyennées par groupe nous ont révélé une variation de $9 \%$ liée à la fréquence du clignotement. L'augmentation d'intensité lumineuse a atténué le ChBF subfovéal de $\sim 32 \%, \sim 30 \%$ et $\sim 5 \%$ respectivement pour la vitesse, le volume et le débit. Par contre, la diminution progressive de l'intensité n'a pas démontré le même effet En général, les données nous indiquent que l'activité générée par les photorécepteurs est associée à un changement de la distribution du $\mathrm{ChBF}$ dans le fond d'œil selon le degré et le type de stimulation des photorécepteurs. 
Mots clés : Electroretinogramme par flash bleu, débit sanguin choroïdien, hémodynamique choroïdienne, clignotement, mesure du débit par laser Doppler, activité neuronale rétinienne. 


\section{Abstract}

The objective of the present study was to reveal an interaction between choroidal blood flow $(\mathrm{ChBF})$ and light-induced photoreceptor activity, a physiological coupling that is already demonstrated for retinal blood flow, but rejected for the $\mathrm{ChBF}$. Ten healthy adults volunteered for this study. A real-time recording near infrared laser Doppler flowmeter was used to quantify the subfoveal $\mathrm{ChBF}$ while the luminance of blue flicker

between $1 \mathrm{~Hz}$ and $64 \mathrm{~Hz}$ was first increased, then decreased by $4.0 \mathrm{log}$ units in $1.0 \mathrm{log}$ unit steps between $0.0375 \mathrm{~cd} / \mathrm{m}$ and $375 \mathrm{~cd} / \mathrm{m}$. In separate testing, flash electroretinograms (ERGs) provided electrophysiological indices of the relative response of shortwave cones and rods to blue light stimulation. Group-averaged, normalized $\mathrm{ChBF}$ measurements revealed that it was modulated by $\sim 9 \%$ by flicker frequency. Increasing the blue flicker luminance from low-to-high attenuated the subfoveal choroidal flow, volume (ChBVol), and velocity (ChBVel) by $\sim 32 \%, \sim 30 \%$, and $\sim 5 \%$ respectively. Decreasing the luminance from high-to-low over the same range had no effect on the subfoveal choroidal hemodynamics. The markedly different effects of reversed directions of change in blue flicker luminance on the subfoveal ChBF were linked to transitions between roddominated and shortwave cone-dominated retinal responses. Collectively, these findings indicate that blue light-induced photoreceptor response is associated with a differential 
distribution of the ChBF across the ocular fundus according to the degree and type of retinal photoreceptor stimulated.

Keywords : Keywords: blue-flash electroretinogram, choroidal blood flow, choroidal hemodynamics, flicker, laser Doppler flowmetry, neural retinal activity 


\section{Table des matières}

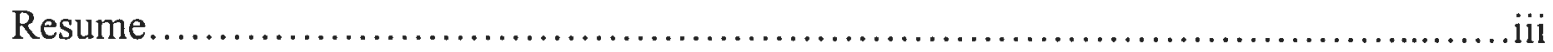

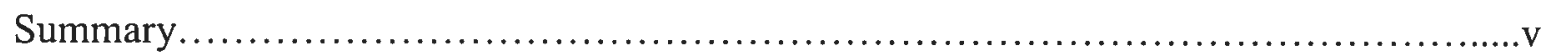

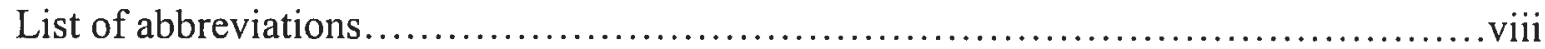

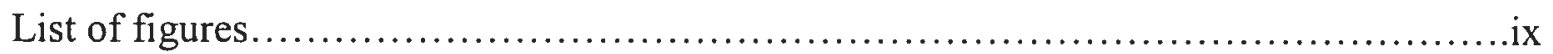

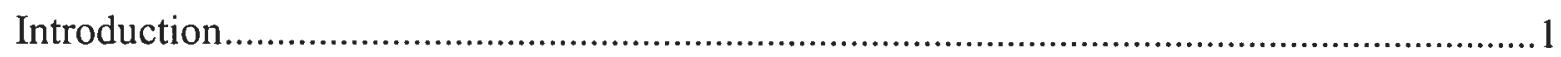

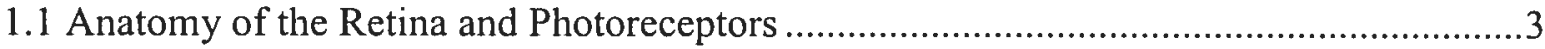

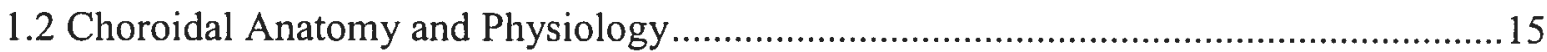

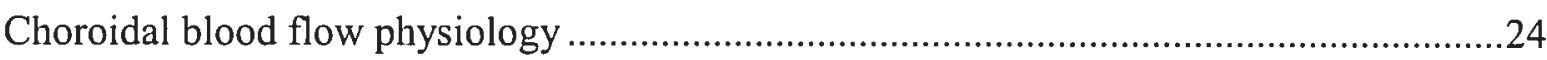

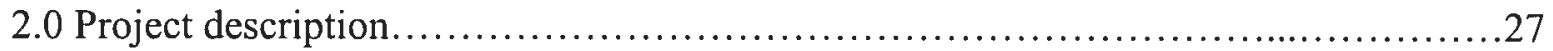

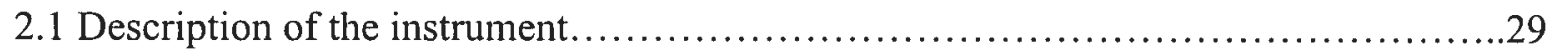

3.0 Materials and methods........................................................

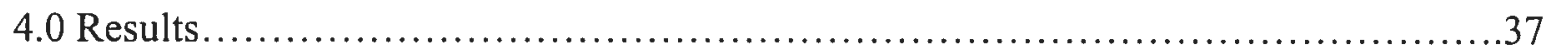

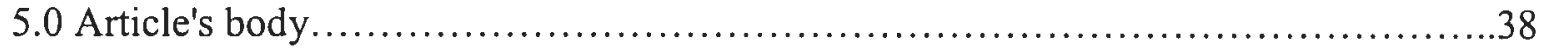

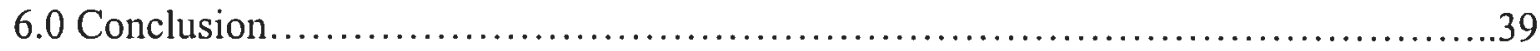

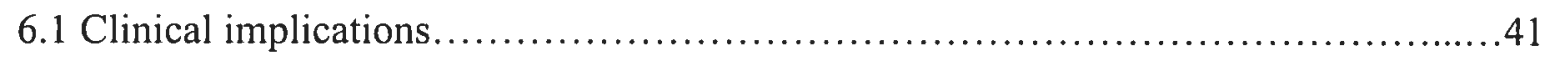

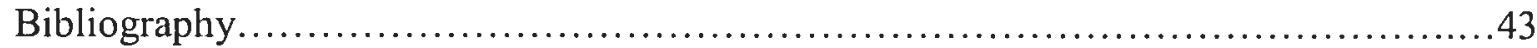

Annexe

Presentation of the flicker stimulator electronic circuit......................... I 


\section{List of abbreviations}

\begin{tabular}{ll}
$\AA$ & Angstrom \\
ANSI & American National Standard For Safe Use of Lasers \\
ARMD & Age Related Macular Degeneration \\
CCD & Charged Coupled Device \\
Cd $\mathrm{m}^{2}$ & Candela per Meter Squared \\
CFF & Critical Flicker Fusion \\
ChBF & Choroidal blood flow \\
DSPS & Doppler Shifted Power Spectrum \\
ERG & Electroretinogram \\
FFT & Fast Fourier Transform \\
Hz & Hertz \\
Khz & Kilohertz \\
LED & Light Emmitting Diode \\
NIR & Near Infrared \\
$n m$ & Nanometer \\
RBC & Red Blood Cells \\
TTL & Transistor-Transistor Logic \\
$V$ & Volt \\
$\mu$ & Micron \\
$\mu \mathrm{F}$ & Microfarad \\
$\Omega$ & Ohm \\
\hline
\end{tabular}




\section{List of figures}

Figure \# 1 Rods and cones distribution by density in the retinal tissue..........4

Figure \# 2 Relative absorbance of photopigments found in rod and cone photoreceptors..................................................... 5

Figure \# 3 Topographic distribution of cones and rods across the retina..........6

Figure \# 4 Waveform of a flash-induced Electroretinogram....................9

Figure \# 5 Intraretinal cell architecture showing connections between cones,rods

bipolar and ganglion cells.......................................... 10

Figure \# 6 Changes in the dark adapted ERG as a function of flash

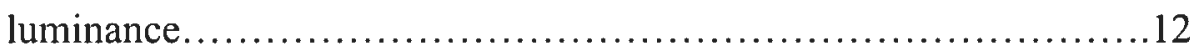

Figure \# 7 Log Spectral sensitivity of rod and cone photoreceptors..............14

Figure \# 8 Retinal view of choriocapillaris at different sites...................16

Figure \# 9 Choroidal Complex........................................... 18

Figure \# 10 Anatomical layout of the lobular sub-units in the choriocapillaris...19

Figure \# 11 Retinal view of choriocapillaris...............................20

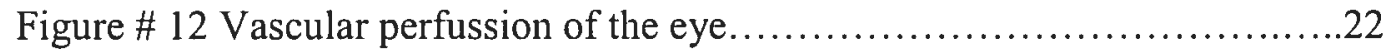

Figure \# 13 Electron microscopy of choroidal and retinal vessel casting........24

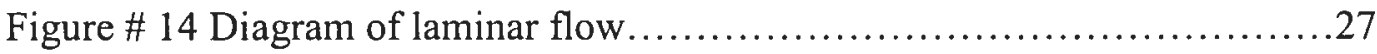

Figure \# 15 Doppler parameters....................................... 31

Figure \# 16 Doppler shift power spectrum................................32

Figure \# 17 Schematic diagram of the laser Doppler flowmeter with the flicker

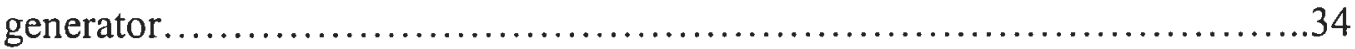

Figure \# 18 Spectral emission of blue flicker stimulator.....................35 


\section{Dedication}

I would like to dedicate this work to my wife Debora who supported me with trust and ecouraged me throughout my life as a source of inspiration. Also to my children : Dafna, Eliel and Abigail who gave me the determination for the important changes in my life.

Finally, to both my parents, Enrique and Ana who supported my education with loving care and inspiration. 


\section{Remerciements}

I would like to express my gratitude to Dr. John Lovasik, for being an outstanding advisor and excellent professor. His constant encouragement, support, and invaluable suggestions made this work successful. His constant dedication to my learning process and love to research encouraged me to enjoy eye research. He has been everything that one could find in a director: knowledge, guidance and friendship.

My sincere thanks go to Dr. Helene Kergoat for her patience and dedication during my training and and also for reviewing my writings.

I also thank Dr. Etty Bitton for having read my research report and provided many important suggestions for improving the accuracy and quality of the thesis presentation. 


\section{Introduction}

The human eye includes an optical system that parallels a photographic camera. Basically, the focusing system is comprised of the cornea and the crystalline lens while the retina serves as the light sensitive interface comprising the light sensitive rod and cone photoreceptors. Retinal light stimulation induces a change in the molecular structure of the photo-labile pigments within the photoreceptors, initiating electrical impulses that travel through the various layers of the retina, along the optic nerve and up to the visual cortex where they are further processed, ultimately leading to visual perception.

The human retina has a very high metabolic rate and as such requires adequate vascular perfusion to sustain optimal neural retinal function. Blood supply to the retina is derived from a dual vascular bed consisting of the choroid, perfusing the outer third of the retina and the central retinal artery circulation perfusing the inner two thirds of the retina. The choroid is one of the most perfused tissues in the human body. The choroidal vasculature represents about $85 \%$ of the total volume of blood perfusing the retina, however, the outer retina extracts only a small percentage (-2-3\%) of the oxygen available from the choroid. The reason for this low oxygen extraction from the choroidal blood flow is not fully elucidated. It has long been hypothesized that this high blood flow level served as a cooling system to prevent the photoreceptors from overheating during light stimulation. However, more recent studies have shown that the retina needed all of the oxygen provided by the choroid to satisfy its metabolic requirements. ${ }^{(15)}$

Vascular autoregulation has been defined as the ability of a living tissue to maintain a relatively constant blood flow despite changes in the vascular perfusion pressure. (Roy and Sherrington ${ }^{(27)}$. From their early experiments on cerebral blood flow regulation, these authors noted that different metabolic products were able to modify the calibre of arterioles and hence blood flow. They proposed that vascular autoregulation was the result of both metabolic and myogenic mechanisms.

It has long been known from studies in animals and humans, that the central retinal artery circulation possesses the capacity to regulate its blood flow during altered vascular 
perfusion pressure or metabolic stress ${ }^{(7)}$. On the other hand, studies have shown that the choroidal circulation did not have the capacity to regulate its blood flow. More recently, however, studies in animals ${ }^{(14)(13)}$,Jeff Kiel et al in studies in pigeons and in humans, Lovasik et al $2003^{(11)}$ and Riva et al $1997^{(25)}$ have demonstrated the capacity of the choroid to regulate its blood flow over a certain range of altered perfusion pressure. Up to now, however, it was believed that blood flow in the choroid was independent of changes in retinal metabolism caused by retinal light stimulation ${ }^{.(7 ; 16 ; 21 ; 22,14,28)}$

The present study was therefore undertaken to evaluate the relationship between choroidal blood flow and retinal metabolism. The principal objective was to determine if and how blood flow is regulated in the normal healthy human choroid in the presence of retinal light stimulation. 


\subsection{ANATOMY OF THE RETINA}

\section{Retina and Photoreceptors}

\section{Photoreceptors}

The retina is a thin three dimensional complex of neurons and glial tissue lining the inner part of the eyeball. It is considered to be part of the brain because during embryonic development it forms as a projection of the forebrain. Within the retina are included the sensory neurons (rod and cone photoreceptors) that respond to light and are connected in an organized fashion to other retinal neurons to form a specialized neural circuit that initiates the first steps of vision at the retinal level.

Histologically, the retina has three principal cells, the photoreceptors, bipolar cells, and the ganglion cells. The photoreceptors are found in the outermost layer of the retina such that light must pass through all retinal layers to initiate photo-transduction. Once light has caused a change in the isomerization of photopigments in the photoreceptors, minute electrical signals are transmitted through the retina by the bipolar and ganglion cells and ultimately reach the visual cortex to initiate visual perception.(18)

In mammalian evolution, retinas have at least two types of photoreceptors: rods and cones. Typically primates and humans have a "duplex retina" allowing good visual discrimination over a wide range of lighting conditions. The number of rods in the retina exceeds those of cones by a ratio of about $20: 1$. The rod free area in the retina known as the fovea corresponds to about 1 degree of visual angle. The absolute number of photoreceptors in the human retina is approximately $120 \times 10^{6}$ rods and about $6 \times 10^{6}$ cones. ${ }^{(12)}$ 
But The region in the center of the retina is called the fovea and contains only cones very densely packed. The cone density rapidly declines with eccentricity from the fovea. The rods begin to appear about 1 degree from the center and their density increases to a maximum at about 17-20 degrees eccentric to the fovea, and thereafter decreased in density towards the far periphery as shown in figure 1 below.

Figure \# 19 Rods and cones distribution by density in the retinal tissue

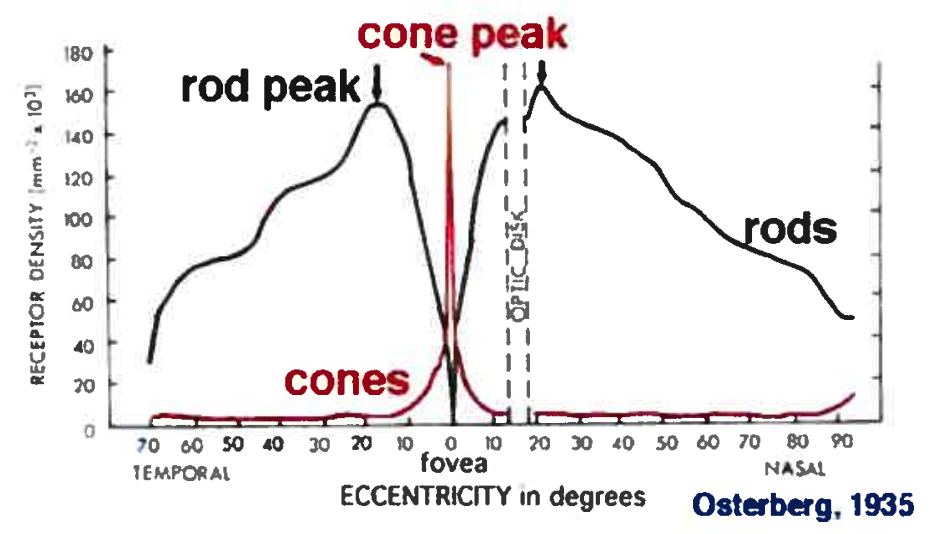

Rod and cone distribution in human retina. Density scale is $10^{3}$ photoreceptors per $\mathrm{mm}^{2}$. Note the absence of photoreceptors in the optic disk.

Rods are highly light sensitive photoreceptors and their principal function is light perception under dim-dark conditions. Rods house a visual pigment called rhodopsin that undergoes photo-isomerization when is activated by a photon of light. The peak spectral sensitivity of rhodopsin is in the region of about $498 \mathrm{~nm}^{(5)}$ 
Figure \# 20 Relative absorbance of photopigments found in rod and cone photoreceptors

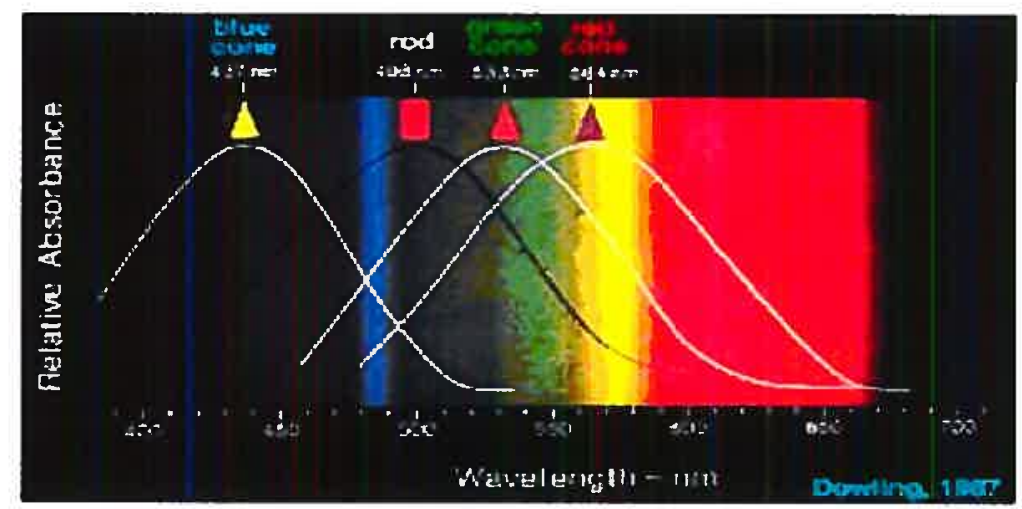

Caption: Normalized view of rods and cones spectral sensitivities for different light wavelengths.(From Webvision by Dowling,1987).

Conversely, cones contain opsins as visual pigments and due to the differences in the molecule of the visual pigment, they are more sensitive to either long wavelengths (red light) with maximal sensitivity at $564 \mathrm{~nm}$, medium wavelengths (green light) with peak sensitivity at $533 \mathrm{~nm}$ and short wavelengths (blue light) with peak sensitivity at $437 \mathrm{~nm}$.

Such long, medium and short-wave cones (L-cone, M-cone and S-cone, respectively) have been found in higher primates as well as in human retinas.

Morphologically, L,M and S-cones differ from each other; S-cones have longer inner segments that go deeply into the inner retinal space . 
Moreover, the number and distribution of cones across the retina is not uniform. L-cones represent $33 \%$ of the population while $M$ - cones peak in the fovea at $64 \%$ and may vary from 52 to $5 \%$ elsewhere in the retina. ${ }^{(12)}$.

The S-cones have their lowest density in the foveal pit representing only $3 \%$ of the cone population to reach a $15 \%$ peak some 1 degree from the foveola (from Latin:central portion of the central retina that contains cones only.) and $8 \%$ of the total population elsewhere in the retina.

The most interesting point is that the signal derived from S-cones are processed differently in the retinal circuitry from $\mathrm{L}$ and $\mathrm{M}$ cones, probably, because the blue system is older in phylogenetic evolution.

Figure \# 3 Topographic distribution of cones and rods across the retina

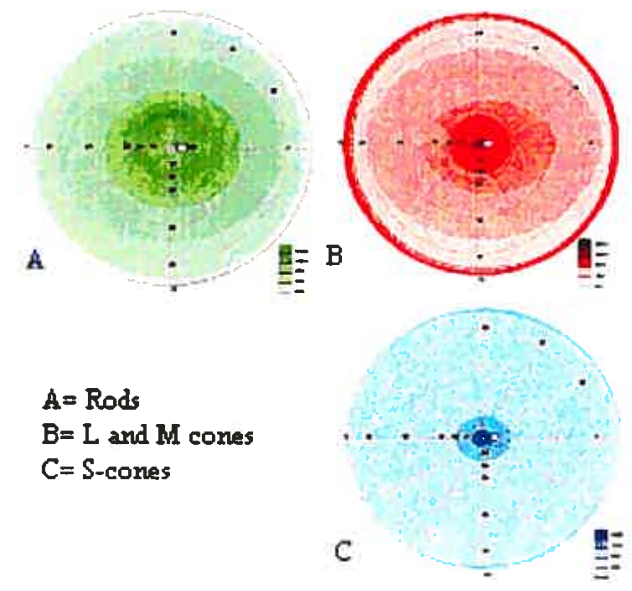

Caption for Fig 3: The rod distribution across the fundus is shown in green. The distribution of medium and long wavelength sensitive cones is shown in orange. The distribution of blue sensitive cones is shown in blue. 
Rods are very sensitive to light in darkness and dim light conditions and they can actually detect one quanta of light under certain viewing conditions. Unlike cones, rods are much slower to respond to light stimulation and electrical signals generated in photopic conditions. may arrive $1 / 10$ of a second later than those originating from cones. ${ }^{(17)}$ Therefore, the rod retinal circuitry with its convergent intermediary neurons is specifically designed to collect and amplify small light quanta for twilight and night vision. ${ }^{(18)}$

The intraocular portion of the optic nerve, referred to as the optic disk is the region where axons of ganglion cells connected with photoreceptors, exit the spherically shaped eye to connect with higher levels of visual processing in the brain As the optic disk is devoid of photoreceptors, any image projected onto this area cannot be seen, and hence is named "the blind spot". ${ }^{(12)}$

Figurel shows that the photoreceptors are symmetrically distributed about the fovea except at the blind spot where there is a complete absence of light sensitive neurons.

In the centre of the fovea, where cones are packed most densely, the neural synapses are displaced to the side giving more direct light stimulation of the photoreceptors. This distribution gives rise to a central depression called the "fovea" (Latin= small pit ).

In the peripheral retina, several rods are capable of synapsing to one ganglion cell, hence, it determines a converging system being able to collect more light under low luminance levels regardless of visual discrimination 
In contrast, the number of ganglion cells corresponding to the foveal region is about equal to the number of cones in a ratio of $1: 1$. Although it is considered that every cone has a direct connection to only one bipolar-ganglion cell, each ganglion cell is nonetheless affected by the actions of a greater number of cones and each cone may influence the activity of several ganglion cells because of lateral connectivity through other retinal neurons typically at the outer and inner plexiform layers. ${ }^{(18)}$

\section{Electrical response of the retina to diffuse light flashes}

The global electrical response of the retina to a diffuse flash of light is referred to as the electroretinogram (ERG). This signal is typically recorded at the corneal level that results from extracellular currents generated by the retinal neurons in response to light activation of the photoreceptors.

Clinically the most important components of the ERG include an electro-negative awave and an electro-positive b-wave. The a-wave results from the hyper-polarization of the photoreceptor membrane after light induce isomerization of the visual pigment which subsequently gives rise to a potential change in the photoreceptors. This process occurs in the outer segment of the photoreceptors. 
Figure \# 4 Waveform of a flash-induced Electroretinogram.

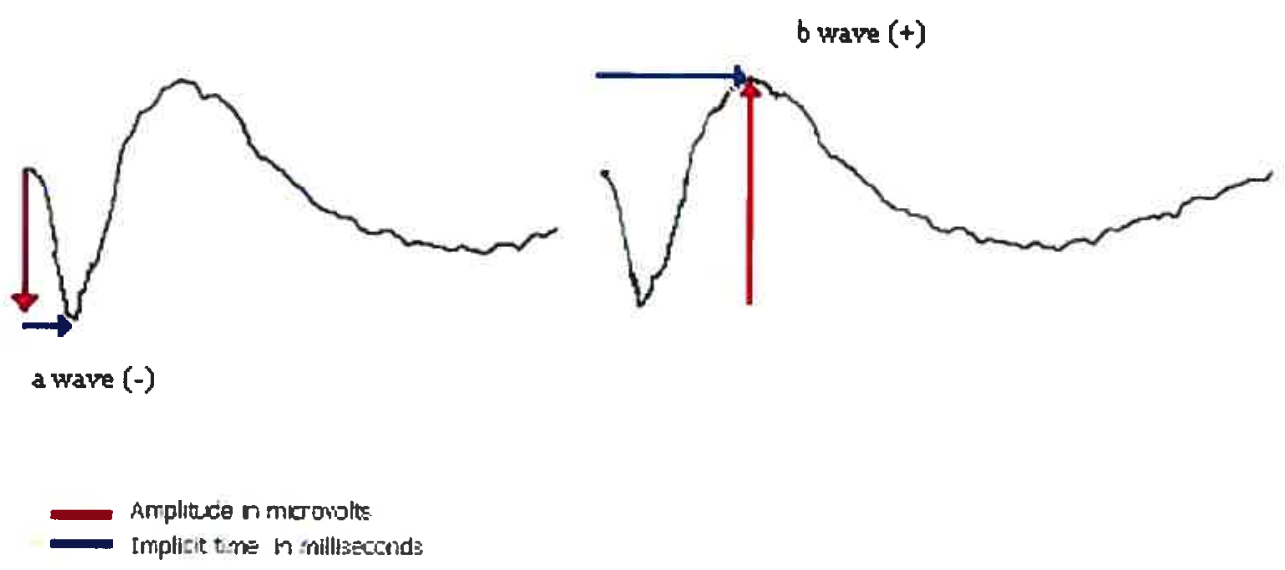

Caption: Typical forms of ERG showing implicit time and amplitude for both $\mathrm{a}$ and $\mathrm{b}$ waves.

The interaction between different cellular types in the primate retina, determines the existence of an eccentrically organized circular receptive fields with center $\mathrm{ON}$ and periphery OFF (and vice versa) synapsed to the ganglion cells and Muller cells.

The $b$ wave is an electropositive deflection receiving its major contribution from the $\mathrm{ON}$ center of bipolar cells and / or the potassium potential difference across the membrane of Muller cells in the retinal circuitry. The time between the onset of a flash and the peak of the b wave is referred to as the "implicit time" and is measured in milliseconds.

Regardless of the origin of the b-wave, it reflects the photoreceptor light response postsynaptically as well as the degree of light adaptation. 
As stated previously, the human retina can be subdivided into two subsystems, the rod system for night vision, and the cone system for day vision, hence a duplex retina. The principal contributors to the flash ERG, photoreceptors, bipolars, and ganglion cells are shown schematically in Figure \#5 below.

The anatomical and physiological complexity of the human retina is reduced to five cell types in clinical electrodiagnostics. These include the photoreceptors (rod and cones), the bipolar cells, the Mueller cells, the amacrine cells and the ganglion cells. Each of these can be functionally isolated to evaluate the structural and functional integrity of the neural layer in which each of these cell type populations are found.

Figure \# 5 Intraretinal cell architecture showing connections between cones,rods bipolar and ganglion cells

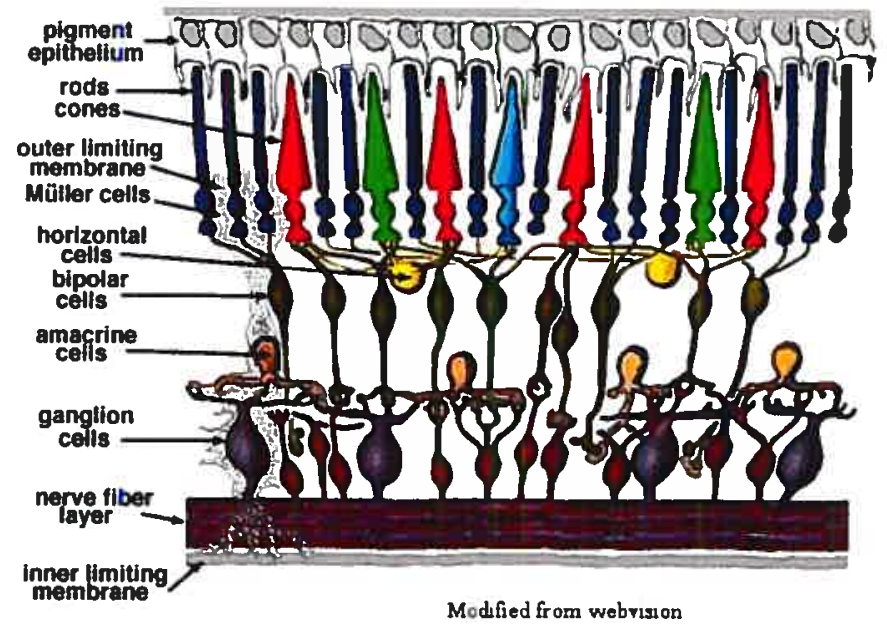


The cone isolated ERG (Photopic ERG) is not as sensitive to dim lights as is the rod -dominated ERG (Scotopic ERG). When a bright light is used to stimulate the retina, cone photoreceptors respond very rapidly (typically within $30 \mathrm{~ms}$ ) with an amplitude that is much smaller than a rod-isolated ERG because of the 20:1 rod: cone ratio. Conversely, if the ERG is acquired under scotopic conditions, the resulting ERG is considerably larger in amplitude but slower to generate the ERG " $a$ " and " $b$ " waves.

\section{$\underline{\text { Scotopic ERGs used as an objective index of retinal response to blue flashes }}$}

In the present study, the scotopic ERG, was used as an objective measure of the global retinal response to flicker of dim-to-bright light.The bright-to-dim blue flashes selectively activated the rods because these contain the pigment rhodopsin that is very sensitive to blue light. Blue sensitive cones have been activated by brighter light flashes.

It is particularly important to note here that the amplitude and timing of the scotopic ERG b-wave was used to show that the retina responded differently when the retina was stimulated by dim-to-bright blue flashes in $1.0 \mathrm{log}$ steps versus bright-to-dim blue flashes in 1.0 log steps over a 4 log range. The differential retinal responses to flashes of identical luminance that were presented in reverse order helped confirm that the measured changes in $\mathrm{ChBF}$ at the fovea reflected the changes in the degree of metabolic activity in topographically discrete segments of the retina. Specifically, when eccentrically distributed rods were increasingly activated by 1.0 log luminance steps, more blood was progressively shunted towards this site of increased retinal activity as indexed by the step-wise decrease in the ChBF at the foveal test site. This stealing of blood in favor of areas of greater metabolism is a common phenomenon in exercise physiology that seems to occur also in the human eye. ${ }^{(11)}$ 
Figure \# 6 Changes in the dark adapted ERG as a function of flash luminance.

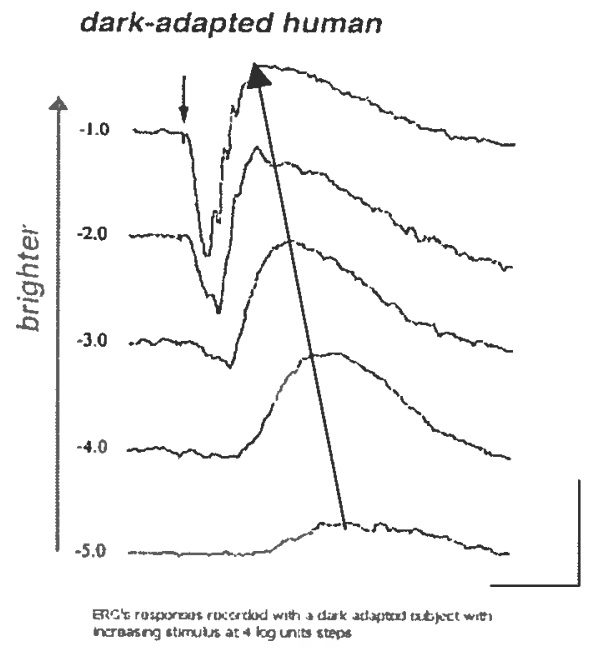

Caption: An increase in the flash intensity causes the amplitude of the scotopic b-wave to increase and the implicit time of the b-wave to decrease. The oblique arrow shows the change in the b-wave implicit time with brighter flashes.

Changing the intensity of the flash reveals that the profile of the ERG changes in two significant ways. First, the amplitude of the b-wave increases with flash intensity. Second, the implicit time of the b-wave decreases with an increase in flash intensity. It is also seen that the a-wave becomes visible at relative flash intensity -2.0 and higher. This is because prior to that stimulus intensity the positive deflection of the b-wave overwhelmed the development of a measurable a-wave.

In contrast to the dark adapted responses, when the retina is sufficiently light adapted, the amplitude of the a-wave increases with flash intensity but demonstrates a constant implicit time. Furthermore, the amplitude of the photopic ERG is significantly 
smaller than the one obtained with a bright flash scotopic ERG because of the large difference in the number of rods versus cones in the human retina

Another important factor is the wavelength of the test flash used to elicit the ERG because the spectral sensitivity curves for a cone vs. rod mediated response differs due to their differential spectral sensitivity. The rod spectral sensitivity curve peaks in the bluegreen region of the visible spectrum at about $500 \mathrm{~nm}$, but the spectral sensitivity for the cones results from the sum of the three subpopulations of short, medium, and long wavelength sensitive cones. Therefore the peak sensitivity for the cone population falls in the green-yellow region of the spectrum at about $560 \mathrm{~nm}$.

The rod system is considerably more sensitive to light than the cones. There is a 3-4 Log difference in light sensitivity favoring the rods over the cones. However, for wavelengths above $620 \mathrm{~nm}$, sensitivity for both photoreceptors becomes similar as shown in fig \#7 
Figure \# 7 Log Spectral sensitivity of rod and cone photoreceptors

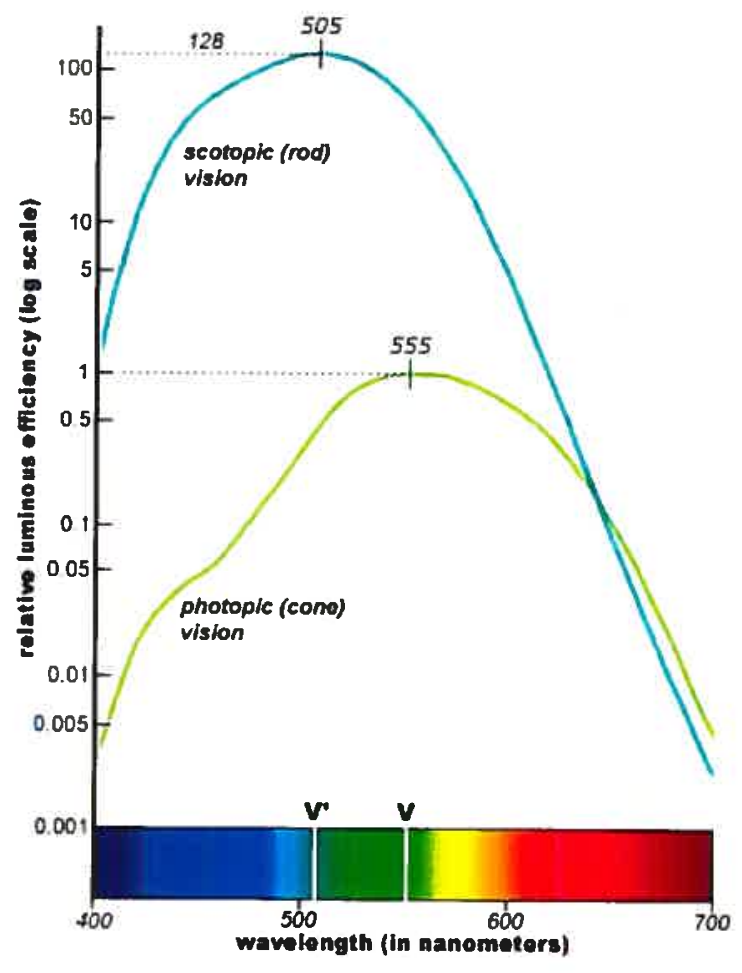

Rod and cone mediated activity can also be separated due to their differential response to light flashes presented across the frequency spectrum.The Critical Fusion Frequency (CFF) represents the highest flash frequency at which a subject just fails to perceive the temporal variation between the light flashes. In photopic conditions, the human eye can perceive flicker up to about $60-70 \mathrm{~Hz}$. In scotopic conditions where the rods dominate the response, the CFF is reduced to flicker levels between $20-25 \mathrm{~Hz}$. (3:17) 


\subsection{ANATOMY OF THE CHOROID}

\section{Choroidal Anatomy and Physiology}

The choroid is a vascularized and pigmented tissue that forms the posterior part of the uveal tract. It extends from the ora serrata anteriorly to the optic nerve posteriorly. The choroid is light to dark brown and it has a spongy consistency. The choroid lies just posterior to the retinal pigment epithelium and is separated from it by Bruch's membrane. It is the only blood supply to the outer portion of the retina and is the sole vascular supply for central vision at the fovea.

The posterior choroid thickness is $\sim 0.22 \mathrm{~mm}$ and 0.10 to 0.15 anteriorly. The choroid is attached to the sclera by strands of connective tissue creating a potential space between them called suprachoroidal space. ${ }^{(10)}$

The innermost choroidal layer is tightly adhered to the retinal pigment epithelium and the optic nerve. The choroidal circulation perfuses both the retinal pigment epithelium and the retina up to the outer aspect of the inner nuclear layer of the retina. Overall, the choroid roughly represents a vascular bed between the junction of the anterior and posterior cilliary arteries (Fig 11).. Furthermore, the density of the choriocapillaris plexus varies roughly with the density of photoreceptors. In the human eye, the choriocapillaris is most dense at the fovea and decreases in density towards the periphery. The variation of the choriocapillaris across several fundus sites is illustrated in Fig 8 below. 
Figure \# 8 Retinal view of choriocapillaris at different retinal sites.

$$
\mathrm{a}=\text { arterioles } \mathrm{v}=\text { venules }
$$

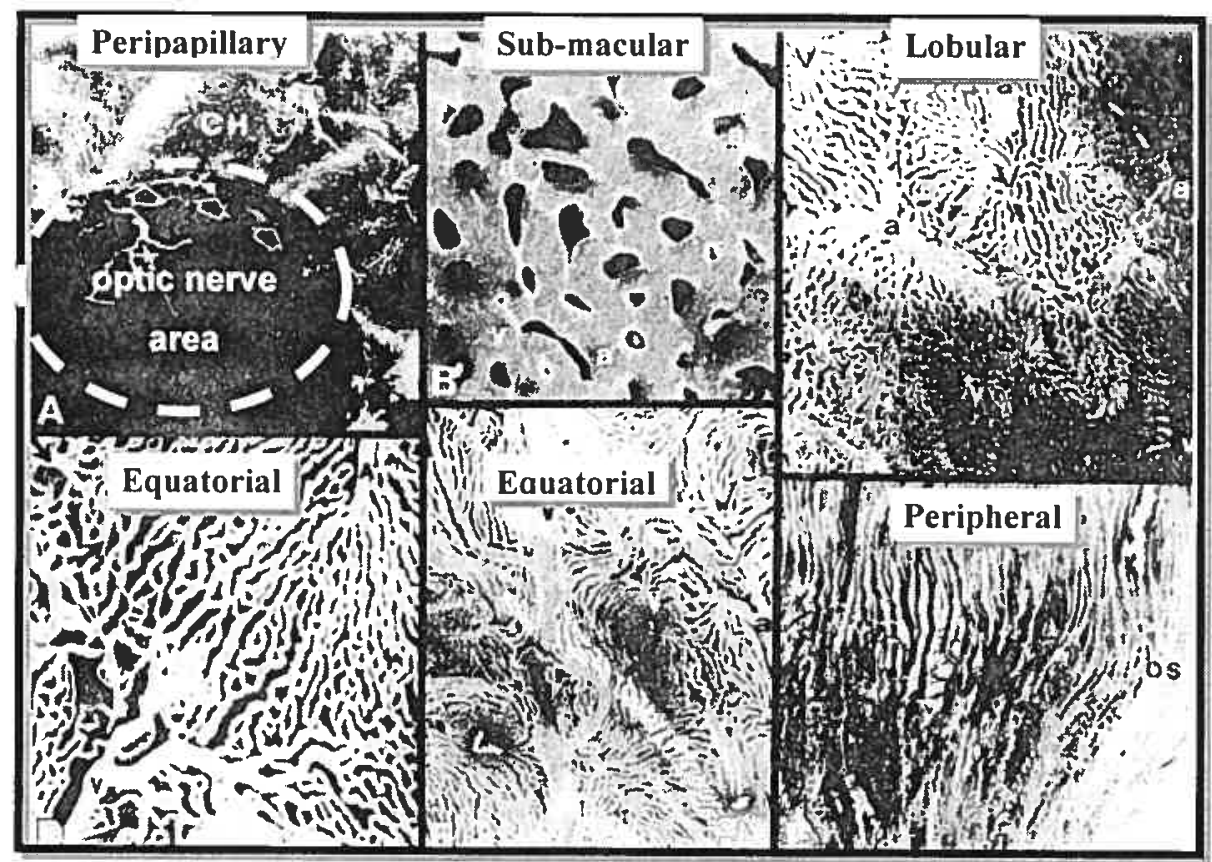

Caption: Human choriocapillaris,posterior pole from retinal view.Montage from peripapillary to peripheral areas. ${ }^{(29)}$

The choroid is considered to be an expandable vascular plexus that supplies nutrition for the rod and cone photoreceptors across all parts of the retina, and also perfuses up to about 130 microns of the outer retina, particularly in the macular area Fig \#12 (choriocapillaris in the subfoveal portion of the retina) 
Anatomically, the outermost layer in contact with the sclera is called the lamina fisca and the suprachoroidal space. Underlying is the Haller's layer characterized by a large number of arterial and venous plexuses. Sattler's layer is the next one containing medium sized vessels and is located in the center of the choroidal complex. (Fig \#9)

The choriocapillaris layer is characterized by large and fenestrated vessels of approximately 40-60 microns in diameter in the same plane of Bruch's membrane. This vasculature offers a large enough diameter to allow several blood cells to circulate simultaneously in any part of the choriocapillaris. The anatomical layout of the choroidal complex with its various vessels as seen from the scleral side is illustrated schematically in Figure \#9 below.

Figure \# 9 Choroidal Complex

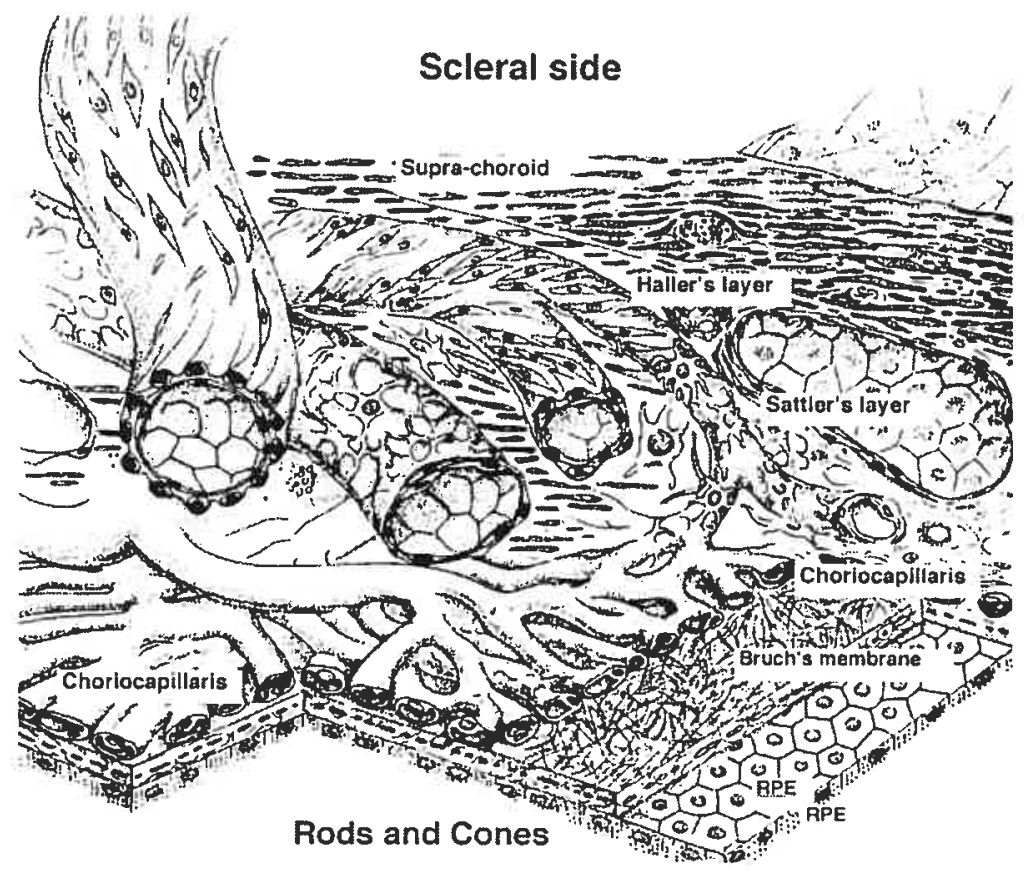


Casts of the choriocapillaris suggest that it functions as an end arteriolar network. In the posterior pole the choriocapillaris has a lobular pattern with a central precapillary arteriole and a peripheral post capillary venule. ${ }^{(9)}$

The anatomical layout of the lobular sub-units in the choriocapillaris from the scleral side is illustrated schematically in Figure \#10 below. The green outline delimits the approximate position of a single lobule in the choriocapillaris. The arterial blood inflow and venous outflow are marked by bold arrows.

Figure \# 10 Anatomical layout of the lobular sub-units in the choriocapillaris

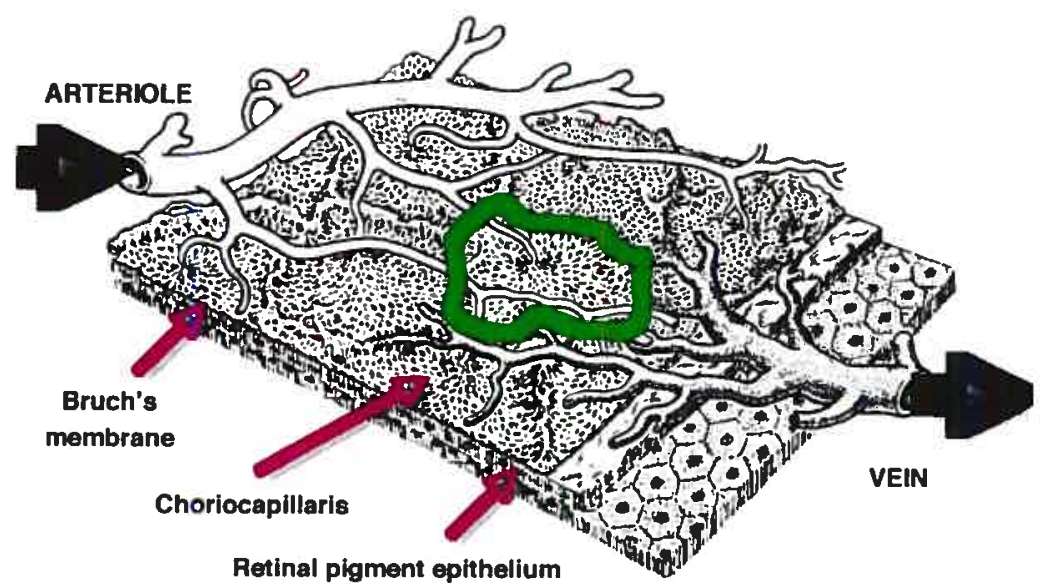

Caption; Diagrammatic representation of the choriocapillaris and its functional organization into roughly hexagonal subunits called lobules, outlined here in green

$$
\text { ink. }{ }^{(9)}
$$

In the present study, non-invasive measurements of choroidal blood flow were made from the retinal side by a near infra red (NIR) probing beam that passed through the retina and penetrated the retinal pigment epithelium to sample blood flow parameters of flow, volume and velocity. Specifically, choroidal blood flow (ChBF) was measured in the 
choriocapillaris of the subfoveal choroid. A picture of a choriocapillaris cast revealing its lobular appearance where blood flow measurements were made in the present study is shown in Fig 11 below. The NIR probing laser sampled the displacement of blood cells in this lobular complex.

Figure \# 11 Retinal view of choriocapillaris

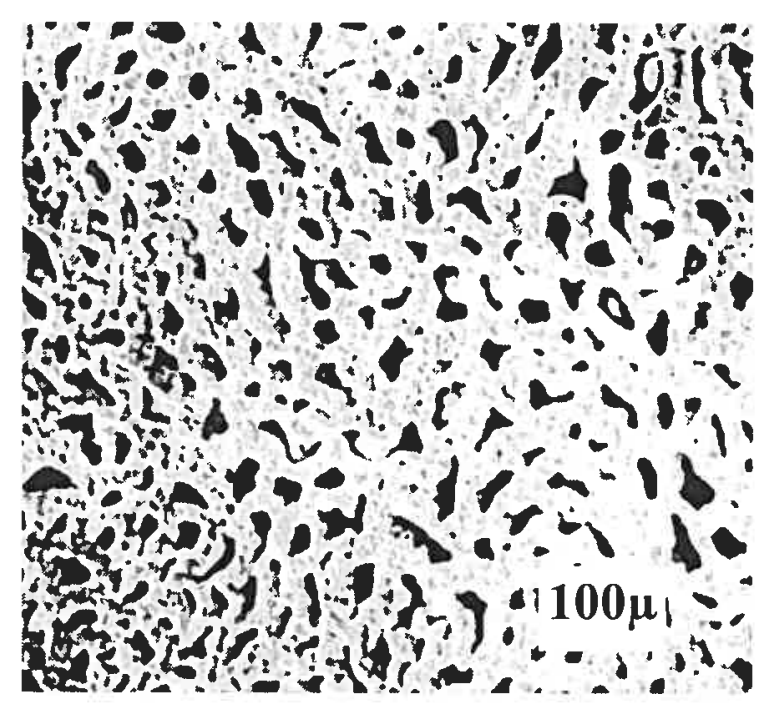

Caption: Anterior view of choriocapillaris in avascular foveal zone. ${ }^{(29)}$

The ophthalmic artery, a branch of the internal carotid artery, divides into a medial and lateral posterior ciliary artery. Before penetrating the sclera both arteries divide into one long posterior ciliary artery and a variable number of short posterior ciliary arteries. The long posterior ciliary artery pierces the sclera some $4 \mathrm{~mm}$ distant to the optic nerve and 
proceeds anteriorly through the suprachoroidal space to subdivide in the area known as the ora serrata into 3 to 5 branches that proceed posteriorly to supply the ora at the equator. ${ }^{(12)}$

The short posterior ciliary arteries pierce the sclera and in the subchoroidal space branch into the choriocapillaris to supply blood flow for the posterior choroid. The insertion point of the short and long ciliary arteries at the posterior pole and their travel in the eye are illustrated schematically in Fig. 12 below (extracted from Wolff's Anatomy of the Eye and Orbit). ${ }^{(4)}$ Note that the short posterior ciliary arteries contribute to the choroid just lateral to the optic nerve head.

The long posterior ciliary arteries, on the other hand, move in the supra-choroidal space and perfuse the ciliary body, iris and the extraocular muscles. One of the four principal drainage vessels, the vortex veins (from latin: vena vorticosa), is shown on the right side of the diagram. Note also, that the choriocapillaris would be the vascular layer closest to the photoreceptors (not illustrated) so that its circulation is vital for sustaining normal retinal metabolism. 
Figure \# 12. Vessels comprising the Vascular perfussion mechanism of the eye.

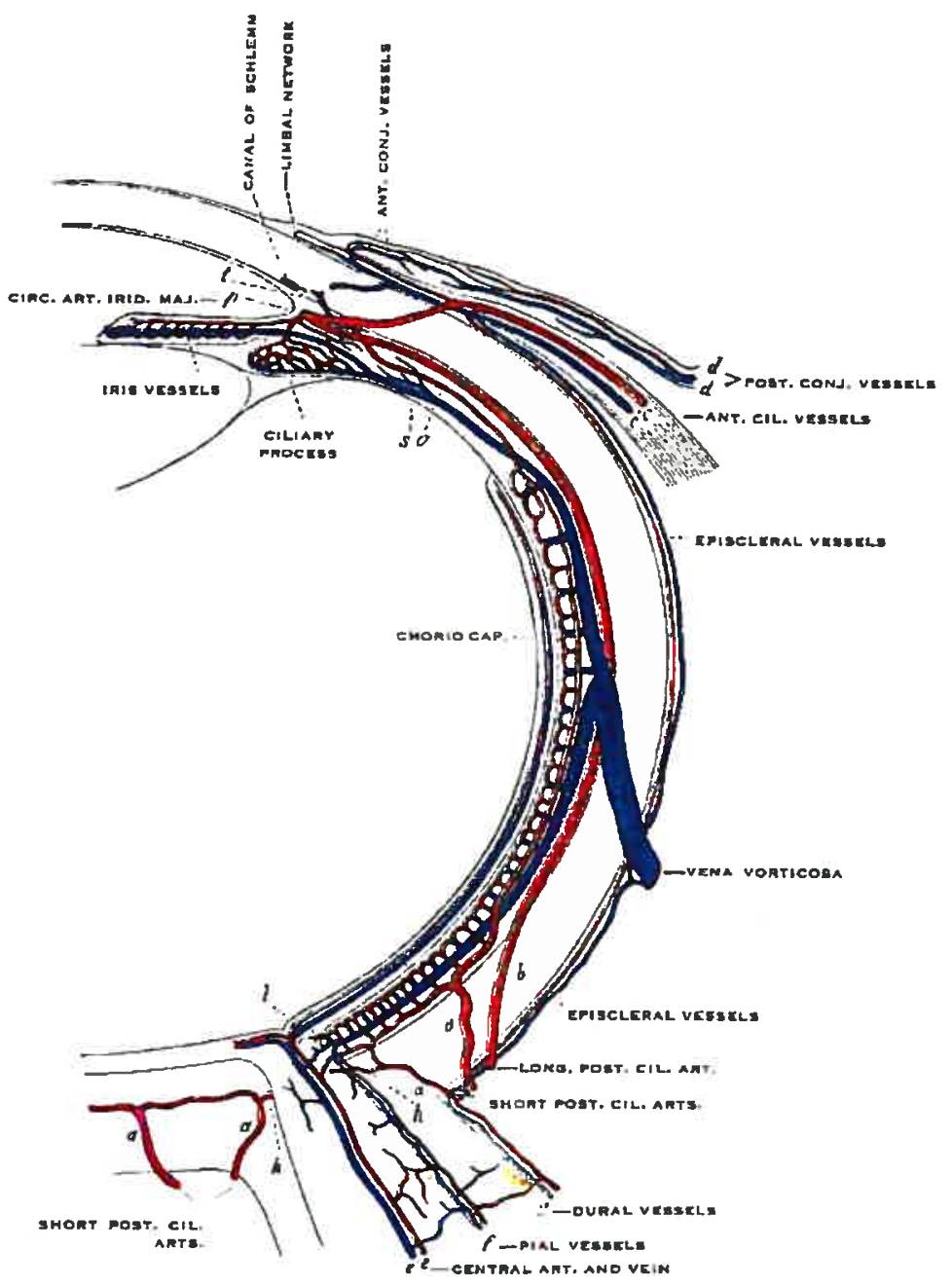

The choroid is also supplied by the anterior ciliary artery accompanying each of the rectus muscles, that mainly supplies the major circle of the iris and provides branches to the ciliary muscle and choroid. Drainage of the choroid is principally via the vortex veins and secondarily by the ciliary body anterior veins. 
Post-capillary venules form afferent veins that provide drainage around the inner sclera. In each of the quadrants, a main vortex vein is connected to these afferent veins via a wide ampulla. Each of the 4 vortex veins in the eye narrows at the end of this ampulla and descends through its respective quadrant of posterior sclera between the rectus muscles. Rarely there may be a secondary vortex vein in the same quadrant. These veins are similar to other small veins with a diameter of about 300 microns. The vortex veins drain blood into the superior and inferior orbital veins. The superior vortex veins pass through the orbital fissure to the cavernous sinus, while the inferior orbital vein goes through the inferior orbital fissure to the pterygoid plexus.

\section{Choroidal innervation}

The choroid receives extensive innervation from both sympathetic and parasympathetic systems. The sympathetic system has a regulatory mechanism through vaso-constriction to keep the blood flow constant, but the parasympathetic system has no effect on $\mathrm{ChBF}$. The choroid is innervated by the short posterior ciliary nerve . They enter the sclera $4 \mathrm{~mm}$ away from the optic nerve and as soon as they reach the suprachoroidal space they lose the myelin and develops arborization. The long posterior ciliary nerves supply the innervation to the anterior choroid branching at the ora serrata level. The long posterior ciliary nerve joins the nasociliary nerve whereas the short posterior cilliary nerve goes through the ciliary ganglion.

\section{Choroidal Histology}

Microscopically the choroid consist of 4 layers. From the outermost these layers are the:suprachoroid,stroma,choriocapillaris and Bruch's membrane. The suprachoroid lays between the pigmented sclera and the large vessel choroidal stroma. It is 30 microns thick 
and has no vessels. Furthermore, it consists of tightly packed collagen fibers and nerve plexus.

The stromal layer consists of blood vessels and collagen fibers. Both arteries and veins are present with major number of veins compared to arterioles. The Haller layer is the most external part consisting of wide diameter arteries and veins, melanocytes and nerve fibers. These vessels are not fenestrated. The Sattler's layer consists of smaller diameter and more intertwined vessels.

Figure \# 13 Electron microscopy of choroidal and retinal vessel casting

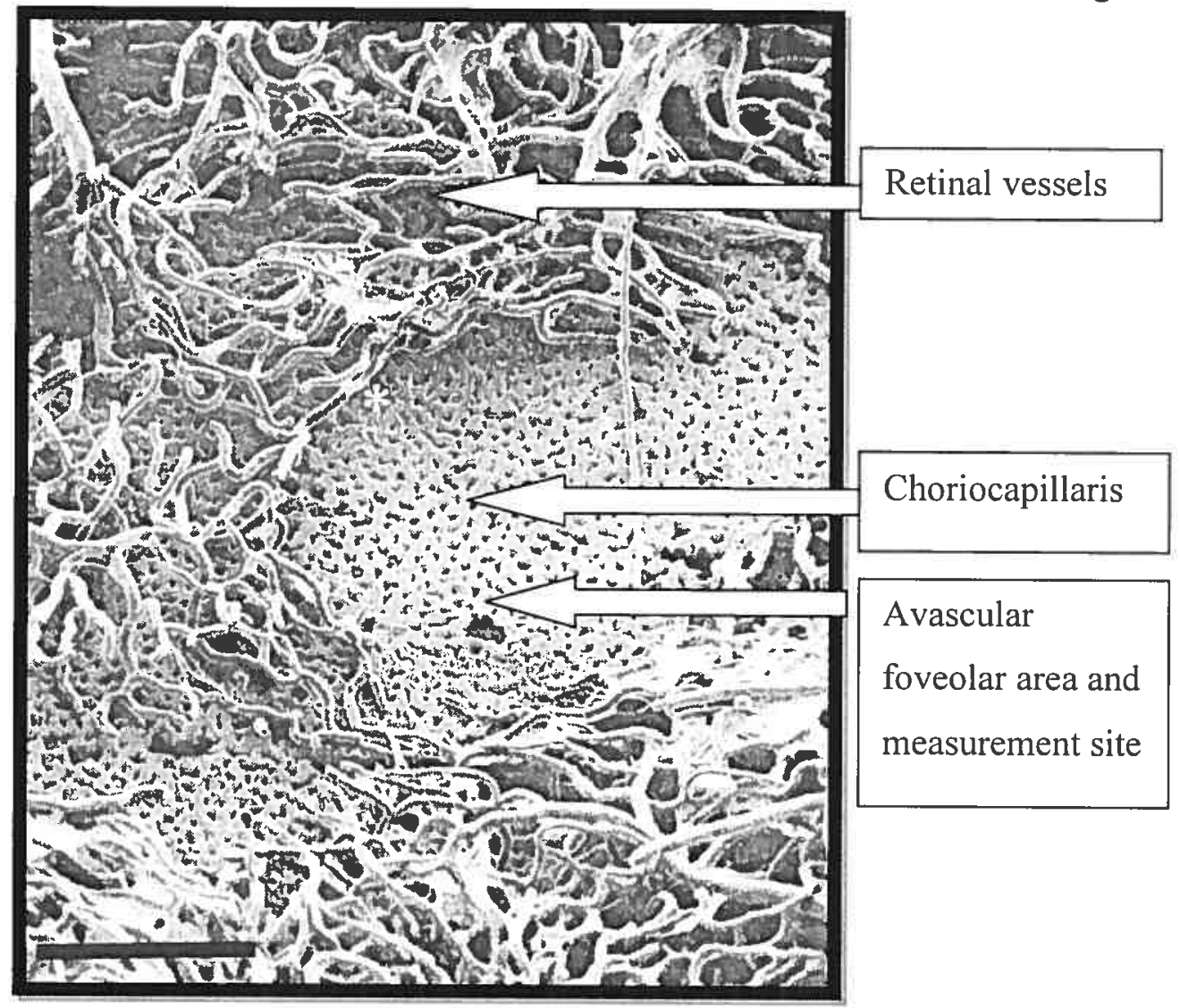

Caption: The foveal area in antero-posterior view.microphotograph shows perifoveal retinal capillaris and underlying choriocapillaris.the diameter of this area measures about 400 microns. $^{(29)}$ 
The choriocapillaris has a unique histological structure crucial to perform its functions. Capillaries in this layer are 40-60 microns in diameter with very thin walls. Vascular walls are provided with $600-800 \AA$ (Angstroms, $1 \AA=1 \times 10^{-10} \mathrm{~m}$.) fenestrations facing the Bruch's membrane with a covering diaphragm to allow metabolites to diffuse to the retina.

This capillary system is characterized by a rapid transformation from arteriole to capillary. Several studies ${ }^{(9)}$ have offered a view of the choroidal vasculature in 3 dimensions to show its unique pattern. In these models, choriocapillaris circulation is explained by a lobular model where a central precapillary feeding arteriole empties into peripherally post capillary venules. This system with many venules provides a rapid blood flow especially in the post equatorial choroid. In the peripheral areas, the arterioles and venules seem to be more parallel to each other interconnecting in a ladder like pattern. The innermost layer is Bruch's membrane or the lamina vitrea. It is a collagenous multilayer of 2-4 microns width that gives support to the retinal pigment epithelium.

\section{Choroidal blood flow physiology}

As mentioned in the section on choroidal anatomy, the choroid is supplied by the ciliary artery branches of the ophthalmic artery. Choroidal circulation is unique because of its lobular anatomy resulting in segmental filling of the choriocapillaris.

Thus blood moves into each lobule and out the relatively low resistance venules. Blood will not be directed to a neighboring lobule because of the relatively high resistance 
compared to the draining venules and this determines a segmental pattern called "watershed zones". (9)

The choroidal blood flow is the greatest in the body and has many times the flow necessary for the nutrition of the outer retina. As part of the incident light on the phortoreceptors is transformed in a small amount of heat, one hypothesis is that this excessive flow protects the retina from over heating when light strikes in acting as a heat dissipator. More recently, the idea of the choroid acting like a heat dissipator has been discounted. ${ }^{(32)}$

This flow is in excess of the oxygen needs of the retinal pigment epithelium and outer retina. This is evident when measurements are made in the artery and vein oxygen concentration difference in the choroidal circulation. This difference comes only to a few percentage points, providing a safety margin in case of flow reduction. However, Linsenmeier has calculated the metabolic needs of the retina and the oxygen content of the choroidal blood and concluded that the retina uses and needs all the oxygen provided by the choroidal blood flow. ${ }^{(15)}$.Choroidal blood flow is under regulation of the autonomic nervous system. Stimulation of sympathetic fibers decreases choroidal blood flow and sympathectomy increases it .

\section{Physics of blood flow}

Blood flow is defined as the total amount of volume passing a point in vessel per unit time. In capillaries the flow of cells and plasma may not be the same. Cells flow faster in the central stream while plasma near the wall moves more slowly. This is referred to as laminar flow. Relative volume of cells and plasma is lower in the capillaries than in larger vessels because cells concentration is higher in central stream in a process named blood skimming. ${ }^{(31)}$ 
Figure \# 14 Diagram of laminar flow

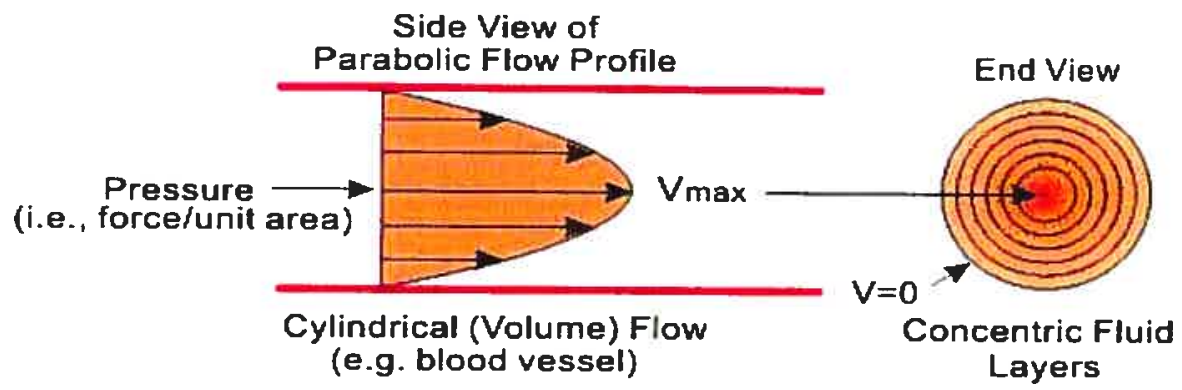

Caption: In laminar flow, fluid speed in the periphery is slower due to friction against the vessel wall. 


\subsection{PROJECT DESCRIPTION}

In the present study, the principal objective was to show that the human choroid was capable of regulating blood flow during changes in retinal metabolism induced by light stimulation of the retina. Blue flicker stimuli were used to specifically activate rod photoreceptors that were topographically isolated from the sub-foveal measurement site of choroidal blood flow. It was clear for us that the appropriate blue light stimulation of the rods would generate a significant change in metabolic demand because of the high number of rods in the human retina, and the high ratio between rods to cones $(20: 1)$.

The underlying hypothesis was that choroidal blood flow would be directed to retinal areas with the greatest metabolic activity. By stimulating eccentrically located rods that are particularly sensitive to blue light because of their rhodopsin content, choroidal blood flow would be directed eccentrically if the hypothesis was correct. In fact, measurements of choroidal blood flow at the fovea revealed a progressive decrease in the subfoveal choroid during increased stimulation of the eccentrically located rod population. This provided direct evidence that the choroid was capable of regulating blood flow.

For many years there was a general agreement that the human choroid was incapable of regulating its blood flow ${ }^{(7)}$. All studies performed in the past were done using invasive techniques such as micro-spheres casting and many of them were not suitable for in-vivo examination. One of the studies was particularly interesting. The authors used lights

of different wavelengths to stimulate the retina of the newborn piglets having retinopathy of prematurity $^{(30)}$. 
By using the microspheres technique they found a significant decrease in the choroidal blood flow when the retina was exposed to blue light whereas the retinal blood flow increased under the same test conditions.

\section{Laser Doppler measurement of choroidal blood flow}

For obvious reasons, we used a system based on an invisible laser probe to detect the moving red cells in the choriocapillaris vasculature. These blood cells reflect the probing beam differently according to the number of moving cells and their velocity. The multiple reflections are detected by a photo amplifier system and the Doppler Shift Power Spectrum produced by the moving particles is produced and analyzed. The resulting data are outputs in relative parameters with respect to blood velocity, volume and flow. 


\subsection{DESCRIPTION OF THE INSTRUMENT TO STIMULATE THE RETINA AND MEASURE CHOROIDAL BLOOD FLOW.}

\section{Materials and Methods}

For the purpose of the present study we needed to measure blood flow with a noninvasive reproducible, accurate and sensitive method to show the changes in the flow, volume, and velocity parameters of blood flow. This method is based on the principle described by the Austrian physicist Christian Doppler in 1842. The Doppler phenomenon describes the frequency shift that a wave undergoes when emitted from an object which is moving towards or away from the observer.

A diode laser module producing a single wavelength of light that is used to detect changes in the frequency of moving particles such as the red blood cells in the choriocapillaris. For the human eye, the Doppler effect can be considered as the change in frequency of a probing laser caused by the velocity of a moving blood cell in a target tissue. Thus, a probing laser with a frequency of $\lambda_{i}$ incident on a red blood cell (RBC) moving at a different velocity $\mathbf{V}$, and an incident laser at an angle of $\boldsymbol{\alpha i}$ will be backscattered at an angle of $\boldsymbol{\alpha}_{\mathbf{r}}$, determines a change in frequency that can be visualized in the following equation:

$$
\Delta \mathrm{F}=\mathrm{n} \cdot \mathrm{V} \cdot\left(\cos \alpha_{\mathrm{i}}-\cos \alpha_{\mathrm{r}}\right) / \lambda_{\mathrm{i}}
$$

In this formula $\Delta \mathrm{F}=$ the Doppler shift.

$\lambda_{\mathrm{i}}$ is the wavelength of the probing laser light within the blood

$\mathrm{n}=$ index of refraction of the light in the blood. 
Figure \# 15 Doppler parameters

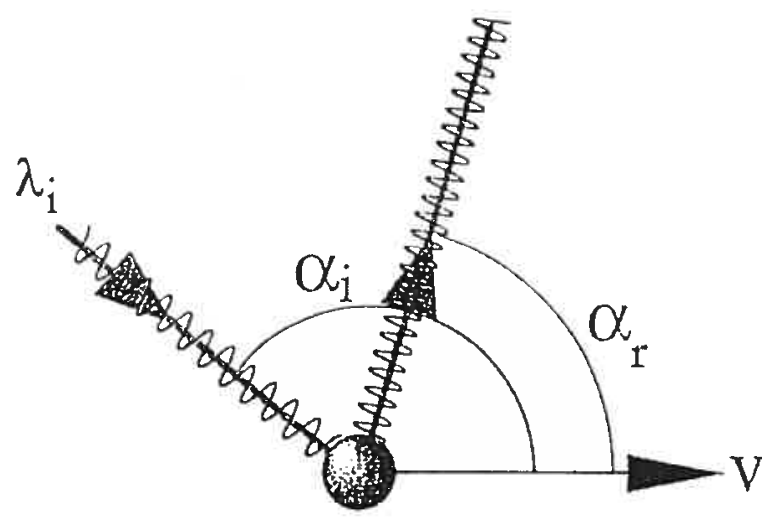

Caption:The incident laser beam $\lambda_{\mathrm{i}}$ is reflected in a moving particle with an angle $\alpha \mathrm{i}$ and reflected in $\alpha_{\mathrm{r}}$ with a different Doppler shifted (From Riva C. et.al.)

When the laser beam strikes the RBC's moving in multiple directions and with different velocities, the backscattered light from the point where the laser is incident on the target tissue represents the summation of multiple waves with various Doppler shifts. The resulting plot of the different shifts versus the Doppler shifted frequency results in the so called Doppler Shift Power Spectrum (DSPS) that defines the frequency proportional to the $\mathrm{RBC}$ movement within a vessel ${ }^{(8)}$. The particle velocity is directly proportional to this difference frequency and can be measured asolutely from the scattering geometry and the laser wavelength ${ }^{(23 ; 24,20)}$

As a typical appearance of such a Doppler shifted power spectrum is presented in Figure 14 
Figure \# 16 Doppler shift power spectrum.

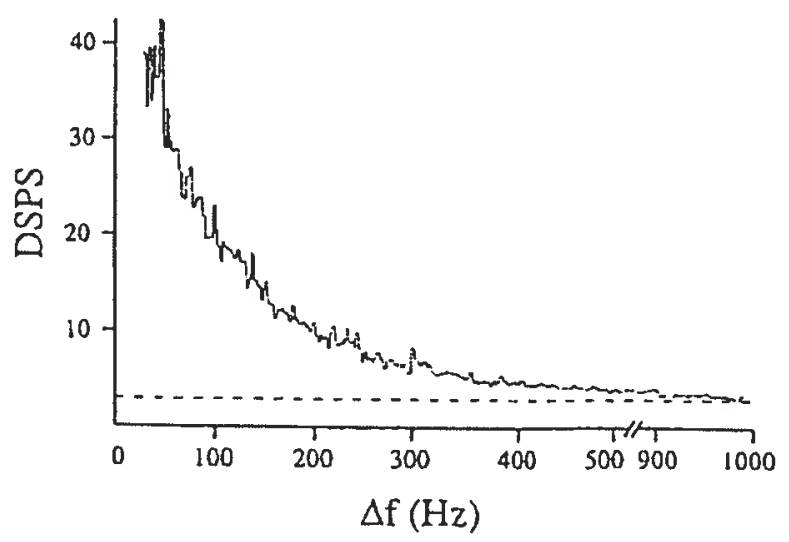

Caption:The backscattered light containing different frequencies acording to the different velocities of the moving particles are shown.dotted line represents the noise threshold level to be filtered.

The horizontal dashed line in this figure represents the "noise" in the measurement system that is omitted during analysis. Typically, elements at the low frequencies that correspond to tissue movements are discarded from analysis. To derive the various blood flow parameters, the area under the DSPS is integrated and processed to yield the following variables:

a) Velocity (Vel): Is measured in kilohertz (Khz) and is related to the difference of the shifted wavelength. This parameter is proportional to the mean velocity of the $\mathrm{RBC}$ 's within the perfused tissue targeted by the laser.

b) Volume (Vol): represents the number of RBC's within the volume of tissue illuminated by the probing laser 
c) Flow: is the result of Vol $\mathrm{x}$ Vel $\mathrm{x}$ constant.

Consequently, the blood flow and volume measured by this near infrared laser Doppler flowmeter system is an extrapolation of the RBC's number and velocity.

It is important to note that the backscattered light also contains light scattered by non-moving elements such as the vessel wall or other supporting structures in target tissues. The frequencies of light backscattered by moving tissue (RBCs) and non-moving tissue are mixed in the process called heterodyning. The intensity of the heterodyned backscattered light undergoes a Fast Fourier Transform (FFT) ${ }^{(19)}$ to yield the Doppler shift power spectrum from which energy related to the heartbeat can be extracted. In this way, light shifted by non-moving tissue is separated from that scattered by moving blood cells.

When this type of analysis is performed in real time, laser Doppler Flowmetry yields a continuous measurement of changes in the above-mentioned blood flow parameters at the measurement site. In the present case, the area of interest was the choroidal blood flow under the foveal area of the retina. This area was selected because it is devoid of retinal blood vessels and thus free from any contaminants related to retinal blood flow.

The laser Doppler flowmeter used in the present study was designed from a Kowa Fundus Camera so as to obtain a Doppler shifted signal while simultaneously delivering flicker stimulation to the retina while measuring blood flow subfoveally. An infrared CCD camera (Pulnix NIR07, USA) was attached to the system to provide a retinal image for each subject to ensure continuous foveal fixation during experimentation.

A specialized flicker stimulation device was placed in the fundus camera light pathway using a 50/50 beamsplitter prism in order to get a coaxial illumination of the fundus over a 30 degree area of the retina. 
Figure \# 17 Schematic diagram of the laser Doppler flowmeter with the flicker generator.

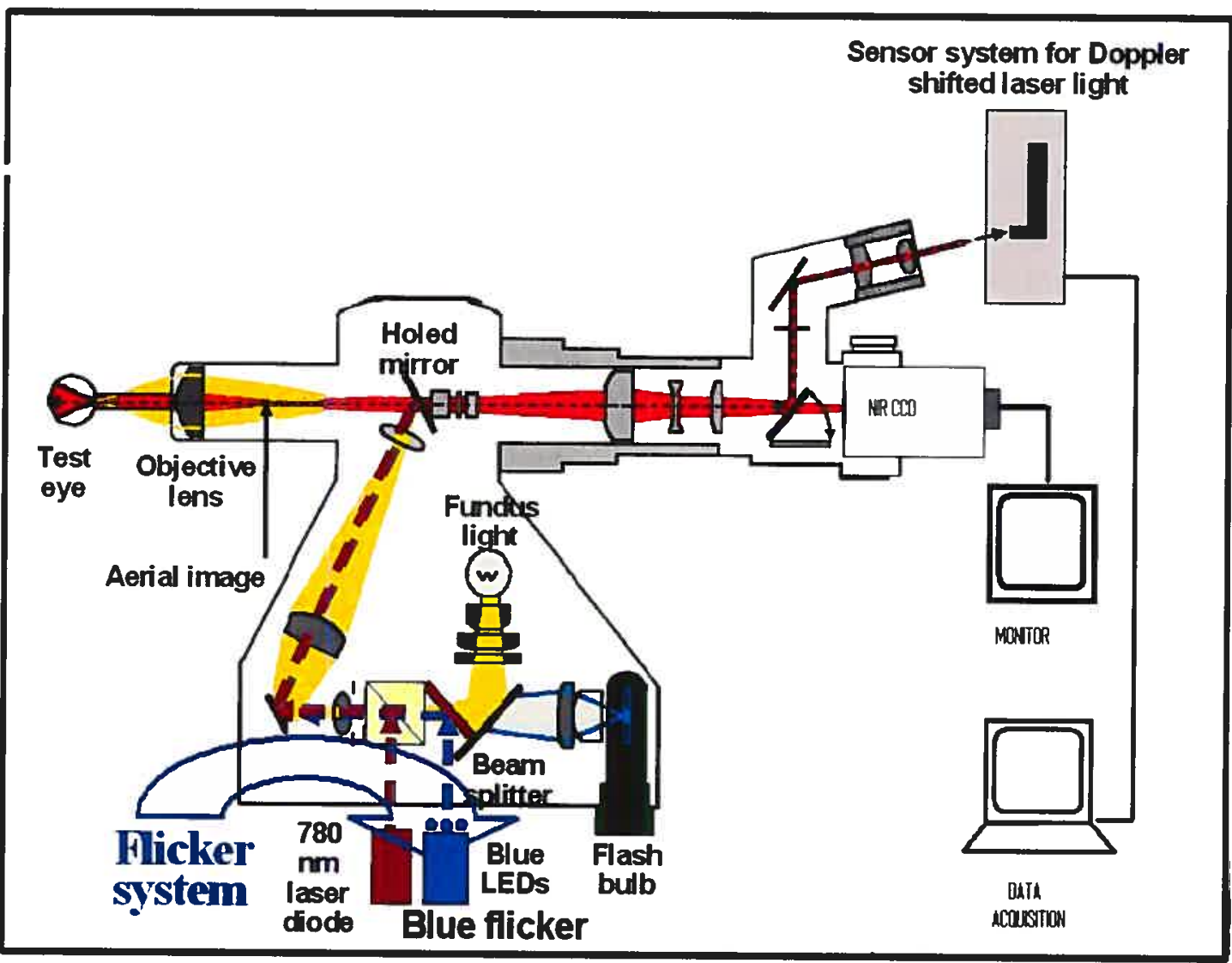

Caption: Fundus camera optic path including the probing laser and the flicker stimulation device.An infrared image of the fundus is detected by the CCD camera and visualized in the monitor.Data is detected by de Doppler head sensor and computer processed.

The probing laser beam was set to an intensity that produced a radiation of approximately 96 microwatts at the cornea with a circular area on the retina approximately 150 microns in diameter. A fiber optics sensor placed on the focal plane of the camera trapped the backscattered light and directed it to a photo detector-amplifier system. The amplifier output was then acquired and digitized in a Next computer using an FFT chip and dedicated software. A FFT analysis was performed in pseudo-real-time (20 measurements 
output was then acquired and digitized in a Next computer using an FFT chip and dedicated software. A FFT analysis was performed in pseudo-real-time (20 measurements per second) while simultaneously recording the heart rate through an earlobe sensor so that the flux from RBC's could be correlated with the systolic- and diastolic phases of each cardiac cycle.

To be able to use various colors of light stimulation in future studies purposes, a four colour (blue, red, green and white) Light Emitting Diode (LED) system was developed and mounted in a rotary carrousel in line with one of the common optical pathways of the fundus camera. However, only the blue flicker channel was used in the present study. The blue LED array consisted of 5 equally spaced LEDs with peak wavelength at $473 \mathrm{~nm}$ and a $22 \mathrm{~nm}$ bandwidth at half peak. The luminance at the corneal plane of the blue flicker light source was $375 \mathrm{~cd} / \mathrm{m}^{2}$ (Minolta CS-100 Chromameter; Milton Keynes UK). By placing neutral density filters (Kodak Wratten, USA) just after the blue LED's, retinal stimulation was modulated over a 4.0 log range in $1.0 \mathrm{log}$ steps.

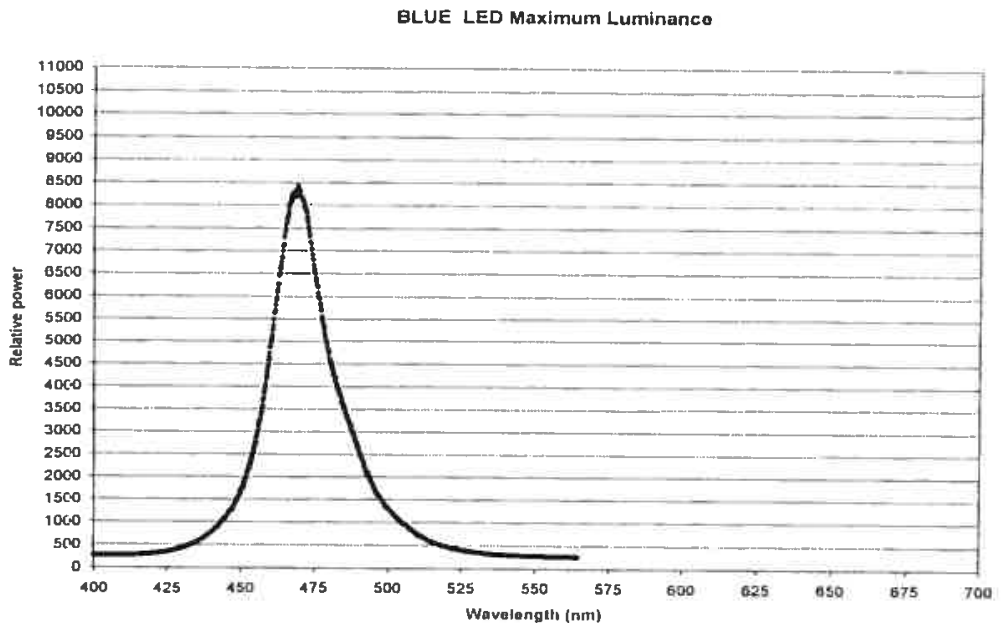

Caption :spectral response of blue flicker stimuli. Peak power is $473 \mathrm{~nm}$ in relative units. 
The flicker stimulator was controlled by a specially built electronic circuit (see Annexe No.1). This circuit allowed delivery of blue flashes at fixed frequencies of 1-2-3-4-5-6-78-9-10-11-12-14-16-18-20-22-24-26-32-44-64 Hz that were modulated on a $50 \%$ on/off duty cycle.

\section{Detection of backscattered light}

The detection of backscattered laser light was detected by the fiber optics sensor placed atop the aerial image of a laser-illuminated spot on the retina. The sensitivity of this light detection system was influenced by two factors:

a) The intensity of the probing Laser light, and

b) The sensitivity of the light amplifier linked with the fiber optics probe.

For the purpose of this experiment we used a near infrared (NIR) $783 \mathrm{~nm}$ laser light. The maximum permissible level of retinal irradiation for a given exposure time increases exponentially with wavelength. Therefore a probing beam in the NIR portion of the spectrum will allow a long safe exposure according to ANSI standards ${ }^{(1)}$ without incurring photic damage. For the case of the $783 \mathrm{~nm}$ laser probe, a single point of the retina could be continuously irradiated for as long as three hours without compromising its safety. The flicker stimulation and LDF recordings in the present study fell well within the established ANSI standards of safety. 
The sensitivity of the detector is crucial to maximize the signal-to-noise ratio of the measurements and still remain within safety levels for the subject with respect to photic damage. Typically an avalanche photodiode is used to detect the backscattered light because of its spectral sensitivity characteristics and it's quantum efficiency for NIR light.

Monitoring neural reactivity of the retina to blue flicker

A clinical signal averaging system (ESPION, Diagnosys;Littleton,MA) was used to quantify the retinal response to flicker at each of the luminance steps used to change retinal metabolism and hopefully evoke measurable changes in the choroidal blood flow. For the purpose of this study, the amplitude and implicit time of the b-wave was quantified and correlated with flash intensity. The ERG recordings were carried out at the beginning and at the end of each flicker series to determine whether the retinal response differed when stimulated by flicker of the same intensity, but presented in a dim-to-bright, vs. bright-todim order. Changes in neural reactivity were then correlated with measurements of subfoveal choroidal blood flow. 


\subsection{Materials and methods This section is described in the article's body}

4.0 Results This section is presented in the article's body. 
5.0 Article 


\title{
Blue flicker modifies the subfoveal choroidal blood flow in the human eye
}

\author{
By
}

Marcelo A. Wajszilber

École d'optométrie

Université de Montréal

Montréal, Québec, Canada 


\section{ABSTRACT}

The objective of the present study was to reveal an interaction between choroidal blood flow $(\mathrm{ChBF})$ and light-induced photoreceptor activity, a physiological coupling that is already demonstrated for retinal blood flow, but rejected for the ChBF. Ten healthy adults volunteered for this study. A real-time recording near infrared laser Doppler flowmeter was used to quantify the subfoveal ChBF while the luminance of blue flicker between $1 \mathrm{~Hz}$ and $64 \mathrm{~Hz}$ was first increased, then decreased by $4.0 \log$ units in $1.0 \mathrm{log}$ unit steps between $0.0375 \mathrm{~cd} / \mathrm{m}^{2}$ and $375 \mathrm{~cd} / \mathrm{m}^{2}$. In separate testing, flash electroretinograms (ERGs) provided electrophysiological indices of the relative response of shortwave cones and rods to blue light stimulation. Group-averaged, normalized ChBF measurements revealed that it was modulated by $\sim 9 \%$ by flicker frequency. Increasing the blue flicker luminance from low-to-high attenuated the subfoveal choroidal flow, volume (ChBVol), and velocity (ChBVel) by $\sim 32 \%, \sim 30 \%$, and $\sim 5 \%$ respectively. Decreasing the luminance from high-to-low over the same range had no effect on the subfoveal choroidal hemodynamics. The markedly different effects of reversed directions of change in blue flicker luminance on the subfoveal $\mathrm{ChBF}$ were linked to transitions between roddominated and shortwave cone-dominated retinal responses. Collectively, these findings indicate that blue light-induced photoreceptor response is associated with a differential distribution of the ChBF across the ocular fundus according to the degree and type of retinal photoreceptor stimulated.

Keywords: blue-flash electroretinogram, choroidal blood flow, choroidal hemodynamics, flicker, laser Doppler flowmetry, neural retinal activity 


\section{INTRODUCTION}

Experimental data pointing to the existence of vascular autoregulation (AR) in the human choroid has been reported only within the last 5 years or so. The principal purpose of choroidal AR is to keep choroidal blood flow (ChBF) constant throughout changes in the ocular perfusion pressure (OPP) such as occurs during exercise. ${ }^{19,28}$ Vascular AR also adjusts blood flow to meet the changing metabolic demands of retinal neurons and to prevent rupture of fine capillary networks where the exchange of metabolites occurs.

The relatively recent adaptation of near infrared (NIR) laser Doppler flowmetry (LDF) ${ }^{26}$ to the human eye made possible several studies into choroidal hemodynamics including the discovery of blood flow regulation in the choroidal vasculature. ${ }^{19,28}$ Unfortunately, the fundamental interaction between light-induced changes in neuro-retinal activity and ChBF has remained largely unexplored.

In lower order animal species, photic stimulation of the retina increases the $\mathrm{ChBF}^{8}$ through the Edinger Wesphal Nucleus reflex. In sharp contrast, diffuse flash stimulation of the human retina has been linked to changes in blood flow in the retinal vasculature and optic nerve head ${ }^{7,10,13,22,24,27}$ but not the choroid. ${ }^{13,18}$ To our knowledge, only two other research groups have examined the interaction between light and $\mathrm{ChBF}$ in man. ${ }^{13,18}$ Longo et al. ${ }^{18}$ were the first to investigate the influence of retinal light levels on the ChBF. Specifically, they found that a 20 minute transition from cone to rod-dominated vision decreased the subfoveal ChBF by $\sim 15 \%$. Subsequent exposure of dark-adapted eyes to ambient room lighting restored the pre-test $\mathrm{ChBF}$ level within 6 minutes. ${ }^{18}$ In as 
much as_the transition from light to dark altered the ChBF, it was surprising that stimulating the central $40^{\circ}$ of the retina for 10 minutes with a bright green light (peak spectral emission at $524 \mathrm{~nm}$, and a $60 \mathrm{~nm}$ bandwidth at $50 \%$ peak transmission) failed to elicit significant changes in the ChBF. However, these conflicting observations could be reconciled if different mechanisms controlled blood flow in the choroid during light-dark exposure of the retina, and when the retina was stimulated by light in photopic conditions.

In a more recent study Garhofer et al. ${ }^{13}$ reported that stimulation of the central $30^{\circ}$ of the retina with $8 \mathrm{~Hz}$ flickering light from a Grass PS2 stroboscope covered with a 550nm low pass filter failed to change the subfoveal $\mathrm{ChBF}$, but did increase blood flow in the optic nerve head by $30 \%$. From these data, Garhofer et al. ${ }^{13}$ concluded that a light-induced increase in retinal activity had no effect on the $\mathrm{ChBF}$, and that the ChBF was independent of changes in retinal metabolism. However, they acknowledged that the $550 \mathrm{~nm}$ lowpass flicker may have preferentially activated the dense cone population at the macula and that the resulting subfoveal choroidal hemodynamics may have differed from ChBF reactions in the greater retinal periphery.

These limited and inconclusive findings on the correlation between light-induced neural activity in the retina and blood flow changes in the choroid prompted us to further investigate this issue. Therefore, instead of using flashes with a broad spectral profile that unavoidably would differentially stimulate all macular cones to different degrees in the macular zone as was the case in previous studies, we presented blue flashes over a broad 
luminance range to selectively stimulate rods and shortwave cones. By presenting blue flicker in a low-to-high luminance order, the goal was to elicit responses principally from the rhodopsin-bearing rods, then the rods and shortwave blue cones, and lastly the shortwave cones with the brightest flicker.

We rationalized that narrowband blue flash $(473 \mathrm{~nm})$ stimulation of the retina at very low luminance was more likely to induce changes at the subfoveal measurement site because of selective activation of rods populating the greater retinal periphery instead of exciting the cones and rods located closer to the fovea by high luminance flicker. Because the rod to cone ratio in the human retina is about $20: 1,{ }^{5}$ we postulated that strong activation of rods by dim blue flashes would change the distribution of $\mathrm{ChBF}$ across the retina and this would be reflected by changes in the subfoveal ChBF. It is likely that previous studies could not find $\mathrm{ChBF}$ changes at the fovea because they used either broadband spectral stimuli, or bright narrowband light that stimulated all cone types in the macula and these determined the metabolic need for $\mathrm{ChBF}$ in the foveo-macular zone.

In order to determine which type of retinal photoreceptor and the degree to which it was activated by the blue flash luminance sequences, retinal responses were compared using the amplitude and temporal features of the flash-evoked retinal potential, the electroretinogram (ERG). 


\section{MATERIALS and METHODS}

Subjects and procedures

Ten healthy adult volunteers, 7 women and 3 men, with an average age of 25.4 years $(\mathrm{SD}: \pm 9.4)$ participated in this study. All participants were experienced research subjects with excellent fixation ability. The subjects' range of refractive errors was $+1 \mathrm{D}$ to $-5 \mathrm{D}$ equivalent sphere, the intraocular pressure (IOP) was between 10 and $20 \mathrm{mmHg}$, and the systemic blood pressure (BP) was within normal limits. All testing protocols complied with the declaration of Helsinki, and ethical guidelines for humans in research at the University of Montreal. A signed informed consent was obtained from each participant after the nature and purpose of the experiment had been explained to each volunteer.

\section{Measurement of choroidal blood flow}

Prior to testing, each subject was familiarized with the experimental protocols. The pupil of the test eye was dilated with 2 drops of MYDRIACYL ( $1 \%$ tropicamide), and one drop of MYDFRIN (2.5\% phenylephrine $\mathrm{HCl}$ ). Choroidal hemodynamics were quantified with a non-invasive continuously recording near infra-red (NIR) 784nm Laser Doppler Flowmeter (LDF; Oculix, Switzerland). All subjects adapted to the normal laboratory lighting for at least 20 minutes prior to any measurements of ocular blood flow. Because the design and operation of the dynamic LDF used to measure the subfoveal ChBF have been reported previously, ${ }^{26}$ only a summary of its use is presented here. The LDF consisted of a fundus camera modified to deliver the narrow laser beam into the foveal avascular zone. The laser power at the cornea was $90-95 \mu \mathrm{W}$ which complied with the 
ANSI 136.1 standards for laser safety. ${ }^{1}$ The laser power was kept constant throughout the entire experiment through a feedback power supply. The laser light back-scattered by static tissue and moving blood cells in the subfoveal choriocapillaris was focused in the retinal image plane of the fundus camera, and a $90 \mu \mathrm{M}$ diameter fiber optics sensor guided the laser light to an Avalanche photodiode. The photocurrent was sampled at $20 \mathrm{kHz}$ and then processed by a dedicated program on a NeXT computer to provide real-time (about 22 points per second) measurements of blood flow parameters based on the Bonner and Nossal theory. ${ }^{3}$ The hemodynamic parameters included the choroidal blood flow (ChBF), velocity $(\mathrm{ChBVel})$ and volume $(\mathrm{ChBVol})$. The measured blood flow represents the product of the blood cell velocity and the concentration of blood cells in the sampled tissue volume. A blood flow sensor clipped to the ear provided measurements of the heart rate throughout the experiment. All LDF measurements were carried out in moderately reduced room lighting to minimize reflections of visible light into the light sensor for the back-scattered light. As a further precaution to eliminate stray light from contaminating blood flow measurements, a $50 \mathrm{~mm}$ dichroic filter ("cold" filter) sealed the aperture leading to the small chamber containing the fiber optics sensor; thus only IR from the test eye was analyzed.

\section{Flicker stimuli:}

A cluster of 5 high intensity blue light emitting diodes (LEDs) with a peak spectral emission at $473 \mathrm{~nm}$, and a $22 \mathrm{~nm}$ bandwidth at half peak height was used as the flicker light source. The first experimental protocol involved increasing the luminance of blue 
flicker in $1.0 \log$ unit steps, with neutral density filters, from $0.0375 \mathrm{~cd} / \mathrm{m}^{2}$ to $375 \mathrm{~cd} / \mathrm{m}^{2}$. In the follow-up experiment, the order of step changes in flicker luminance was reversed. The blue flicker frequency and stimulus duration were controlled by dedicated electronics. Light from both the LEDs and the NIR laser was projected onto the retina by the background illumination optics of the fundus camera. An auxiliary optical system directed the probing laser light into the center of the flicker field that filled the entire field of view of the subject. Maxwellian optics in the fundus camera assured a bright uniform illumination of the retina.

\section{Effect of increased flicker luminance on the $\mathrm{ChBF}$}

This phase of the study involved flicker stimulation of the retina at frequencies between $1 \mathrm{~Hz}$ and $64 \mathrm{~Hz}$ in 24 steps. The duration of each flicker frequency was 10 seconds with no delay between frequencies. A complete ChBF stimulus-response profile involved continuous measurement of the subfoveal $\mathrm{ChBF}$ during 5 consecutive flicker frequency series as described above and presented over a $4.0 \mathrm{log} \mathrm{cd} / \mathrm{m}^{2}$ luminance range. The luminance of the aerial image of the LED matrix formed by the fundus camera optics was $375 \mathrm{~cd} / \mathrm{m}^{2}$ (Minolta CS-100 Chromameter; Milton Keynes, UK).

In this test phase, flicker luminance started at the lowest level, $0.0375 \mathrm{~cd} / \mathrm{m}^{2}$, and was increased to the brightest one, $375 \mathrm{~cd} / \mathrm{m}^{2}$, in $1.0 \log$ unit steps. The lowest flicker luminance, $4.0 \log$ unit steps below $375 \mathrm{~cd} / \mathrm{m}^{2}$ was achieved by placing 4.0 ND Kodak Wratten in front of the LED light source. At the end of each flicker series, normal room lighting was restored for one minute to insure that the retina remained light adapted. 
During this interval subjects maintained fixation of the probing laser, ChBF recordings were stored to computer memory, and the ND filters were changed to increase the flicker luminance by $1.0 \log$ unit step. As such, each test eye underwent 20 minutes of flicker stimulation throughout this phase of the experiment.

Since the LDF system measured only relative changes in choroidal hemodynamics, the $\mathrm{ChBF}$ for all test conditions was recorded in one continuous session to be able to quantify absolute changes in the $\mathrm{ChBF}$ as elicited by changes in flicker frequency and luminance.

\section{Effect of decreased flicker luminance on the $\mathrm{ChBF}$}

Six of the original 10 subjects participated in this follow-up study where the order of flicker frequencies was maintained but the order of step changes in luminance was reversed, such that the first flicker frequency series was presented at a luminance of 375 $\mathrm{cd} / \mathrm{m}^{2}$ and the luminance of each subsequent flicker series was decreased by a $1.0 \mathrm{log}$ unit step down to $0.0375 \mathrm{~cd} / \mathrm{m}^{2}$. It was hypothesized that any cumulative effect on the retina caused by diffuse blue flicker would be changed radically by a reversal in the step changes in flicker luminance. Conversely, if retinal activity and ChBF were unaffected by photic stimulation per se, then a reversal of the step changes in flicker luminance would also have no effect on retinal activity or choroidal perfusion and consequently the $\mathrm{ChBF}$ response profile would be the same whether the flicker luminance was increased or decreased. 


\section{Electroretinographic index of neuroretinal responses to light}

Blue flash (peak wavelength $=473 \mathrm{~nm}$ ) ERGs were recorded to differentiate the contribution of rods and shortwave cones to changes in the $\mathrm{ChBF}$ during blue flicker stimulation of the retina at different luminance levels. The ERGs were recorded with an Espion (Diagnosys LLC, Littleton, MA, USA) evoked potential system respecting ISCEV guidelines for recording clinical ERGs. ${ }^{21}$ Each subject was positioned in the LDF system for ERG recordings. To record the ERG, a DTL fiber electrode ${ }^{6}$ placed in the inferior sulcus of the test eye served as the active electrode, while a second DTL electrode placed in the inferior sulcus of the contralateral light-occluded eye served as the reference electrode. That eye was occluded by a light-tight black patch. $\mathrm{An} \mathrm{Ag}-\mathrm{AgCl}$ electrode on the inner wrist formed the electrical ground. All ERGs were recorded in response to $1 \mathrm{~Hz}$ diffuse blue flashes delivered through the background channel of the fundus camera as during LDF recordings. Each retinal response to a flash was gated through a $0.15-100 \mathrm{~Hz}$ band-pass filter and amplified X 10,000. The average of 10, 200 ms epochs synchronized to the onset of each flash comprised the ERG signal. This ERG was measured before and after each blue flicker series to quantify any changes in retinal responsiveness induced by the flicker. For the needs of this study, the ERG response was quantified by measuring the amplitude of the b-wave. Changes in rod/cone contributions to the ERG b-wave were derived from light-induced changes in the overall ERG waveform, including changes in the amplitude and temporal attributes of the principal ERG components with changes in blue flash intensity.

\section{Data analyses:}


The specific response parameter sought and the various methods used to extract the information from the large database are listed below:

A) Quantifying any changes in choroidal hemodynamics caused by flicker between $1 \mathrm{~Hz}$ and $64 \mathrm{~Hz}$ and step increments in flicker luminance followed by flicker with step decrements in flicker luminance. (see Fig. 1 and 2)

The ChBF response for a particular flicker frequency was averaged over the corresponding 10 seconds flicker interval using an electronic averaging cursor in the LDF program. This process simultaneously yielded the time-averaged value for the $\mathrm{ChBF}$, $\mathrm{ChBV}$ ol, and ChBVel that were then normalized so that blood flow responses across subjects could be compared on a common scale. The $\mathrm{ChBF}$ was normalized by assigning a $100 \%$ value to the 10 seconds of $\mathrm{ChBF}$ recorded in response to the first flicker stimulus (i.e. $1 \mathrm{~Hz}$ ) and luminance level, and expressing subsequent $\mathrm{ChBF}$ responses to incremental changes in flicker frequency as a percentage of the $100 \%$ reference value across all flicker luminance levels. The same data reduction was applied to the ChBVol and ChBVel data for each subject for flicker of increasing and decreasing luminance. Any separation of consecutive flicker frequency response profiles would be due to step changes in flicker luminance.

B) Quantifying the effects of step increments in flicker luminance on the ChBF (see Fig. 3)

While the group averaging procedure was particularly effective for revealing the effect of flicker luminance on the frequency driven response profile, the interaction between flicker frequency per se and the $\mathrm{ChBF}$ was much less evident. In fact, the first analysis 
somewhat obfuscated the frequency effects because the ChBF peaked in narrow but different frequency ranges across subjects. Consequently, a second analysis of raw data was carried out and presented the modulation amplitude for $\mathrm{ChBF}$ as a function of the peak driving frequency.

C) Quantifying the interaction between the choroidal Velocity, Volume and Flow when flicker luminance was increased and then decreased (see Fig. 4)

In order to better quantify the interactions between the Velocity, Volume and Flow, all individual normalized responses for Volume and Velocity $(\mathrm{n}=24$ each) for each flicker luminance level $(n=5)$ were plotted against the corresponding normalized change in Flow for flicker of increasing and decreasing luminance. Subsequently, curve-fitting functions helped define interactions between these hemodynamic parameters.

D) Quantifying changes in retinal sensitivity during repeated exposure to blue flashes of increasing and decreasing luminance (see Fig. 5)

The ERG b-wave peak time and amplitude were measured with electronic cursors. The most revealing and compelling information about the retinal sensitivity status came from a side-by-side comparison of the ERG profiles taken before and after each flicker series on the same scale and over flash luminance series that a) increased luminance and b) decreased luminance.

E) Correlating the Neural and Vascular Responses to graded blue flashes (see Fig. 6) 
The existence of any physiological correlation was explored by simply plotting the normalized change in the subfoveal $\mathrm{ChBF}$ and the absolute ERG amplitude as a function of the blue flash luminance for flashes of increasing and decreasing intensity.

\section{$\underline{\text { Statistical analyses }}$}

ANOVAs on the group-averaged changes in $\mathrm{ChBF}, \mathrm{ChBVol}$, and ChBVel were analyzed with flicker frequency, peak frequency and luminance as repeated measures for an alpha level of $5 \%$. Tukey(a) post hoc comparisons were used where appropriate.

\section{RESULTS:}

Effects of increased blue flicker frequency and step increments in luminance on the ChBF The normalized, group-averaged subfoveal $\mathrm{ChBF}, \mathrm{ChBVol}$, and $\mathrm{ChBVel}$ are presented as a function of flicker frequency and flicker luminance in Figures $1 \mathrm{~A}, \mathrm{~B}$, and $\mathrm{C}$ respectively.

\section{-Fig 1 about here-}

The flicker luminance values used in this phase of the study $\left(0.0375\right.$ to $\left.375 \mathrm{~cd} / \mathrm{m}^{2}\right)$ are presented adjacent to each $\mathrm{ChBF}$ response curve. The vertical bar through each data point in this figure and all subsequent figures in this report represent standard error of the mean (SEM) values for a given data point. The curves drawn through the group averaged data points for choroidal Flow, Volume, and Velocity were determined by fitting polynomial expressions for visible trends, first for points in the low flicker frequency 
range $(1-22 \mathrm{~Hz})$, and then through remaining data points in the middle to high flicker frequency range $(22-64 \mathrm{~Hz})$.

The ChBF results shown in Fig. 1A revealed 2 small peaks in the low frequency range (small inverted arrows) and a more gradual positive peak in the higher frequency range at $\sim 50 \mathrm{~Hz}$ suggesting photic driving of the $\mathrm{ChBF}$. However, these group-averaged peaks did not achieve statistical significance $(p>0.05)$.

Even more visible than the small $\mathrm{ChBF}$ peaks within each of the flicker frequency series illustrated in Fig. 1A was the rather large downward shifts in successive flicker frequency series. Each drop in the $\mathrm{ChBF}$ flicker frequency response profile occurred after a $1.0 \log$ unit step increase in flicker luminance. With each 1.0 log unit step increment in flicker luminance the $\mathrm{ChBF}$ was attenuated by about $8 \%$, except between steps \#3 and \#4. Overall, repeated blue flicker stimulation of the retina across five flicker frequency series and four $1.0 \mathrm{log}$ unit step increments in flicker luminance caused the subfoveal ChBF to decrease from its pre-stimulus value by $\sim 32 \%(p=0.0001)$.

A visual comparison of the population trends for choroidal Flow, Volume and Velocity in Fig. 1 revealed that the overall pattern of change in the ChBF was closely paralleled by the changes in the ChBVol ( $p=0.0001)$ (Fig. 1B) but not in the ChBVel $(p>0.05)$. This trend identified a drop in ChBVol as the dominant hemodynamic parameter that determined the change in the subfoveal $\mathrm{ChBF}$. 
Effects of increased blue flicker frequency \& step decrements in luminance on the ChBF

The normalized, group averaged $\mathrm{ChBF}, \mathrm{ChBVol}$, and $\mathrm{ChBVel}$ response profiles to five consecutive flicker series coupled with four, $1.0 \mathrm{log}$ unit step decrements in luminance are presented in Fig. 2, frames A, B and C respectively. A cursory examination of these data sets reveals that all parametric response curves were clustered more tightly than those of Fig. 1, and that the Velocity response curves shown in Fig. 2C straddled the $100 \%$ reference line. Statistical analyses of the data trends in Fig. 2 revealed that none of the hemodynamic parameters changed during flicker frequency testing that involved step decrements in flicker luminance ( $p>0.05)$. Thus, the reversed order of changes in flicker luminance did not show a large decrease in the $\mathrm{ChBF}$ as seen in increased flicker luminance.

\section{-Fig 2 about here-}

\section{Effects of increased flicker frequency per se on the subfoveal ChBF}

Because raw LDF recordings for each subject showed considerably larger perturbations in the $\mathrm{ChBF}$ than that indicated by the small group-averaged peaks at $\sim 5 \mathrm{~Hz}, \sim 17 \mathrm{~Hz}$, and $\sim 50 \mathrm{~Hz}$, another analysis procedure was used. Specifically, the raw data was re-analyzed and plotted to show the ChBF modulation amplitude for each subject as a function of the flicker frequency eliciting the largest change in the ChBF. The results of this secondary analysis are presented in Fig. 3 and show that the ChBF was preferentially modulated by blue flicker at $\sim 7 \mathrm{~Hz}, \sim 22 \mathrm{~Hz}$, and $\sim 47 \mathrm{~Hz}$ by amounts of $\sim 9 \%, \sim 10 \%$ and $\sim 8 \%$ respectively. 


\section{-Fig 3 about here-}

The vertical and horizontal bars through each of the three data points in Fig. 3 represent \pm $1 \mathrm{SEM}$ for the modulation amplitude and $\pm 1 \mathrm{SEM}$ for the peak frequency driving the changes in the ChBF amplitude, respectively. Statistical analyses showed that these amplitudes of modulation in $\mathrm{ChBF}$ were significant $(\mathrm{p} \leq 0.011)$, but that the $\mathrm{ChBF}$ modulation amplitudes did not differ across flicker frequencies ( $p>0.05$ ). It is noteworthy that the modulation amplitude identified by this second analytical approach was about three times greater than that shown in Fig. 1A and more closely reflected the amplitude of flicker-induced changes seen in the ChBF recordings.

The differential effect of flicker frequency and flicker luminance on the ChBF was particularly noteworthy. Fig. 3 shows that blue flicker frequency between $1 \mathrm{~Hz}$ and $64 \mathrm{~Hz}$ increased the ChBF by a maximum of about $9 \%$. In contrast, the data in Fig. $1 \mathrm{~A}$, as described above, shows that an increase in the flicker luminance $\left(0.0375 \mathrm{~cd} / \mathrm{m}^{2}\right.$ to $375 \mathrm{~cd} / \mathrm{m}^{2}$ ) continued to have an effect on the choroid over a much broader range of luminance than flicker per se, reducing the subfoveal ChBF over 4.0 log unit steps.

Interaction between the choroidal blood Velocity, Volume and Flow as flicker luminance was increased and then decreased

In as much as the subfoveal $\mathrm{ChBF}$ remained unchanged during repeated blue flicker stimulation of the retina when the luminance of flicker was decreased over four $1.0 \mathrm{log}$ 
unit step decrements in the flicker luminance (Fig. 2), the reversal of the order of flicker luminance stimuli was hypothesized to be the reason for the relative constancy of the subfoveal choroidal hemodynamics.

The effect of the order of presentation of blue flicker with increasing vs decreasing luminance on choroidal hemodynamics are compared in the same subgroup of subjects in Fig. 4A and 4B. Fig. 4A shows the systematic reduction recorded in the ChBVol when the flicker luminance was systematically increased over $4.0 \log$ unit steps. In contrast, the ChBVel initially decreased by just $4 \%$ while the volume had decreased by about 10 $\%$. Thereafter, the velocity formed a plateau with its remaining data points.

In contrast to this large decrease in the ChBVol caused by blue flicker with increasing luminance, blue flicker with decreasing luminance produced very different response profiles for the choroidal volume and velocity data. Both the ChBVel and ChBVol clustered tightly between the 90 and $106 \%$ data intervals. A regression line drawn through the velocity data points showed a slight positive trend as did a regression line through the volume data. The large difference in the flicker luminance induced changes in the volume and velocity parameters for choroidal blood flow seen in Fig. 4A and 4B were attributed to the difference in the order of luminance presentation.

\section{-Fig 4 about here-}

Empirical evidence that the same photic stimuli presented in a forward (dim-to-bright) and reversed (bright-to-dim) order to the retina can elicit different retinal responses was 
verified experimentally through objective measurements of light-evoked retinal activity. The results of diffuse $1 \mathrm{~Hz}$ fERGs acquired in response to blue flashes of increasing and decreasing intensity (Plate A vs Plate B) are presented in Fig. 5. These objective indices of neural responses of the retina indicate that the same blue flash intensities when presented in the forward $v s$ reverse direction elicited b-waves with significantly different amplitudes. This evidence suggests a mechanism whereby blood flow at the fovea maybe attenuated in favor of supporting more active photoreceptors in the retinal periphery during repeated blue flicker stimulation of the retina. In a subsequent analysis, the ERG b-waves were measured and plotted as a function of blue flash luminance for test conditions where the luminance increases from dim-to-bright (Fig. 6A) and decreases from bright-to-dim (Fig. 6B). These were then matched with the simultaneously measured changes in the subfoveal $\mathrm{ChBF}$. This latter analysis showed that increasing flicker luminance elicited increased amplitude ERGs and a decrease in the subfoveal ChBF. When the flicker luminance was presented from bright-to-dim, the same flicker luminance elicited different ERG b-wave amplitudes before and after the flicker frequency series. The subfoveal ChBF did not change with flicker luminance.

-Fig 5 about here-

-Fig 6 about here-

When the flicker luminance was increased from $0.0375 \mathrm{~cd} / \mathrm{m}^{2}$ to $37.5 \mathrm{~cd} / \mathrm{m}^{2}$ the ERG bwave increased in amplitude and its peak time shortened (Fig. 5A). This type of change in the ERG b-wave is characteristic of a rod-dominated retinal response. When the flash 
intensity was increased further to $375 \mathrm{~cd} / \mathrm{m}^{2}$ the amplitude of the b-wave was abruptly reduced and its implicit time was shortened, indicating that the rod-dominated response had shifted to a cone-dominated ERG. The overlapping of the waveforms in Fig. 5A revealed that ERGs measured before and after each flicker series were identical and thus no change in retinal sensitivity was induced by the preceding flicker frequency series.

Fig. 5B shows the ERGs before (identified by small letter "b") and after (identified by small letter " $a$ ") each flicker series where the blue flash luminance was decreased from $375 \mathrm{~cd} / \mathrm{m}^{2}$ to $0.0375 \mathrm{~cd} / \mathrm{m}^{2}$ in $1.0 \mathrm{log}$ unit steps. The ERGs before and after flicker were similar for all flash luminances except for the 3.75 and $0.375 \mathrm{~cd} / \mathrm{m}^{2}$ flashes where both retinal potentials were about $50 \%$ smaller than the ERGs elicited by the same flicker luminance but presented in reverse order. The small oblique arrows identify the global retinal response just after the eye had been adapted to room lighting, and before the blue flicker frequency series was initiated. This light exposure would have reduced the rod contribution to the ERG. However, after the retina was stimulated by the blue flicker frequency series, the ERG response increased thereby indicating a larger participation of rods in the recorded ERG. When the blue flash was at its dimmest level, the ERGs before and after adaptation to room light were identical and almost extinguished. These changes in the ERG profiles differed markedly from those elicited by the same light stimuli but presented in reverse order. 


\section{DISCUSSION}

Choroidal blood flow response to flicker

To our knowledge, direct driving of the ChBF by light stimulation of the retina had not been reported previously by other groups for the human eye. In fact, the linkage between $\mathrm{ChBF}$ and a light-activated increase in retinal metabolism had been specifically rejected. ${ }^{13,18}$ These latter reports were not consistent with the notion that the human retina requires all the oxygen provided by the choroid. ${ }^{17}$ If the choroid could not increase the availability of oxygen to the retina during photic stimulation by increasing $\mathrm{ChBF}$, such stimuli might not be processed normally for lack of metabolites, and other ongoing physiological processes could also be compromised. The first report of an association between light-induced neuroretinal activity and changes in ChBF was provided by Lovasik et $a l .^{20}$ This group reported that the amplitude of both flash ERGs and the subfoveal $\mathrm{ChBF}$ increased during red flicker stimulation of the retina. The present follow-up study is the first to provide a detailed report on the systematic effects of light flashes modulated in luminance and temporal frequency on the subfoveal ChBF in the human eye.

\section{Anatomical and physiological evidence for differential blood flow requirements}

Early psychophysical studies established that the spectral sensitivity of the retina some $7^{\circ}$ from the fovea was best described by overlapping scotopic and photopic luminosity functions that peaked at about $500 \mathrm{~nm} .{ }^{14}$ The observation that the greater quantal sensitivity to light of rods over cones increased with distance from the fovea matched the anatomical increase in rod density toward the retinal periphery. ${ }^{14}$ The blue-sensitive s- 
cone population is minimal in the fovea, occurs in highest density in an annulus $2.5^{\circ}$ to $7^{\circ}$ wide about the fovea, half this density outwards to $20^{\circ}$ and a further drop off beyond to the far periphery. ${ }^{2}$ This distribution of rods and s-cones led to our proposal that the 473 nm dim-to-bright flicker series preferentially increased neural retinal activity in the rod populated periphery and that this heightened neural activity could direct $\mathrm{ChBF}$ in favor of the retinal periphery. The progressive decrease in subfoveal $\mathrm{ChBF}$ during blue flicker is consistent with this interpretation. The absence of blood flow changes at the fovea for bright-to-dim flicker was consistent with the $\mathrm{ChBF}$ being driven by the s-cone population that dominated the foveal-macular zone. Such s-cone dominated activity for the highest blue flicker luminance $\left(375 \mathrm{~cd} / \mathrm{m}^{2}\right)$ is supported by psychophysical studies that showed the rod system sensitivity to be reduced by several log units at this light level, and conversely, the cones to be insensitive to very low light levels. ${ }^{14}$ To our knowledge, there have not been any previous reports of a physiological reallocation of $\mathrm{ChBF}$ in the human eye. We believe that these data are the first to reveal this unique capability of the human choroid.

The notion of a topographically specific increase in blood flow to meet the demands of a focal increase in retinal metabolism has been advanced in animal studies. Kiryu et al. ${ }^{16}$ reported that $488 \mathrm{~nm} 8 \mathrm{~Hz}$ flicker increased retinal blood flow in baboons by about $30 \%$, more so in the mid-peripheral region where ganglion cells and nerve fibers were found in greater density. This was interpreted as a regulatory response to meet the increased metabolic demands at discrete retinal sites, similar to zones of increased retinal activity induced by the graded luminance flicker used in the present study. The possibility of 
similar regulatory responses in the choroidal vasculature of sub-human primates was raised by Fryczkowski ${ }^{11}$ who distinguished 2 models of choroidal lobuli, anatomical $v s$ functional, where the latter could modify flow according to metabolic need. In a followup study, Flower $e t$ al. ${ }^{9}$ also reported that lobular filling of blood in the choriocapillaris could be altered by acute elevations in the IOP, and by oxygen and carbon dioxide breathing. Thus, the $\mathrm{ChBF}$ is not merely a passive network of vessels but can be modulated according to regional metabolic needs. Therefore, the anatomical and physiological bases for our proposal has already been reported in sub-human primates. Consequently, the progressive decrease in the subfoveal $\mathrm{ChBF}$ during increased flicker luminance (Fig. 1A) and the observation that the flicker caused the volume of blood to decrease (Fig. 1B) is consistent with this differential choroidal perfusion across different retinal zones.

Such preferential perfusion of metabolically active tissue is well known in functional MRI studies of the brain that consistently show blood moving preferentially towards metabolically active sites. ${ }^{23,25,29}$ For the human eye, however, this proposal demands experimental validation possibly by simultaneous $\mathrm{ChBF}$ measurements in foveal and eccentric regions of the fundus during flicker. The technology to realize dual $\mathrm{ChBF}$ measurements at different retinal sites is yet to be developed.

If one rejects the notion that a preferential increase in rod activity draws blood away from the foveal-macular zone and into the retinal periphery it would have to be assumed that the human eye can withstand a $30 \%$ drop in the $\mathrm{ChBF}$ and volume and still maintain 
normal physiological function. Such a large decrease in retinal perfusion would not only impair retinal function ${ }^{15}$ but would also have effects on overall vascular hydrodynamics thereby making this argument untenable.

\section{Photoreceptor activity and choroidal vascular tone}

The present data show discrete changes in choroidal hemodynamic parameters throughout blue flicker stimulation of the retina. When the percent change in the velocity and volume parameters were correlated with the percent change in choroidal flow during blue flicker stimulation, the results indicated that the change in volume was responsible for the decrease in the subfoveal ChBF (Fig 4A).

In as much as this trend for the volume was seen in all test subjects, we propose that vasoconstriction was the mechanism by which $\mathrm{ChBF}$ was reduced at the fovea. The overall effect of a regional vasoconstriction would likely be a displacement of blood towards the periphery for greater perfusion of a large stimulated rod population. Further, it may be hypothesized that the reduced $\mathrm{ChBF}$ at the fovea returns to pre-stimulus value by the reverse process involving vasodilation where blood from the periphery is returned to the foveo-macular zone. This notion is yet to be verified experimentally.

\section{Correlation between retinal activity and choroidal blood flow}

This light-induced change in the ChBF (Fig. 1) was not found in previous studies likely because of the broad spectral nature of the test flashes. ${ }^{13,18}$ The successful modulation of the subfoveal $\mathrm{ChBF}$ in the present study was due to light stimuli that first stimulated the peripheral rods at very low flicker luminance, then the rods and s-cones at higher 
luminance, and finally the s-cones at maximal flicker luminance. These changes in $\mathrm{ChBF}$ were clearly linked to electrical indices of global retinal function as indexed by diffuse blue flash ERGs. Figure 5 shows that the amplitude and timing of the ERG components were modulated in proportion to the blue flash luminance. Together these data sets show a clear association between neuroretinal activity and blood flow in the choroid.

In an earlier study using the cat model, Buerk et al. ${ }^{4}$ reported flicker-induced changes in blood flow in the optic nerve head as well as changes in the ganglion cell axons that comprise the optic nerve. This relationship between blood flow and neural activity has been coined "neurovascular coupling". In the present study, direct measurements of rod or cone activity by invasive probes were not possible for obvious reasons. However, in clinical neurophysiology, it is well established that the early negative potential wavelet referred to as the a-wave of the flash ERG originates from the photoreceptors that are specialized sensory neurons containing light-sensitive pigments. In this context, our findings can also be interpreted as a form of neurovascular coupling. However, "neurovascular coupling" as a general descriptor of the phenomenon reported here is precluded by the observation that flicker of decreasing luminance (Fig 5B) elicited changes in the ERG but not in the subfoveal ChBF.

The observation that reversing the step changes in flicker luminance had no effect on the subfoveal $\mathrm{ChBF}$ across flicker luminance (Fig. $2 \mathrm{~A}$ ) is consistent with the notion of $\mathrm{ChBF}$ being directed to retinal zones with highest neuronal activity. The ERG records in Fig. 6 revealed that before each flicker set, the global retinal response was much smaller than 
that for the same flash luminance but presented in the forward (dim-to-bright) direction. As such, less $\mathrm{ChBF}$ would have been needed to support an attenuated rod dominated response; consequently, the subfoveal $\mathrm{ChBF}$ would be minimally affected (Fig 6B), as verified empirically during the reversed luminance flicker study (Fig 2A).

Referring to Fig. 5A, the diffuse blue flash ERG b-wave elicited by the dimmest luminance level $\left(0.0375 \mathrm{~cd} / \mathrm{m}^{2}\right)$ was relatively flat and delayed. As the flash intensity was increased, the ERG amplitude increased rapidly to its largest and quickest value for a $37.5 \mathrm{~cd} / \mathrm{m}^{2}$ flash after which it decreased by about $50 \%$ and showed the fastest response time to flashes of $375 \mathrm{~cd} / \mathrm{m}^{2}$. For all these test conditions but the last, rods were progressively recruited into the response. For the $37.5 \mathrm{~cd} / \mathrm{m}^{2}$ blue flash, shortwave cones also contributed to this response while the smallest and fastest ERG was a shortwave cone-dominated ERG. The progressive recruitment of rods by blue flashes of increasing luminance was presumed to require increased choroidal perfusion in the periphery where rods occur in greatest density.

The ERG response profiles in Fig. 5A and 5B show that the same flash luminance elicited different retinal responses depending on whether the test luminances were increased (Fig. 5A) or decreased (Fig. 5B). Parallel to these objective indices of retinal function, the $\mathrm{ChBF}$ also responded differently when the flicker luminance increased or decreased. Thus, the argument that a singular stimulus should elicit the same retinal response is untenable. Clearly, the preceding retinal light history strongly influences the retinal response to a light flash with fixed spectral and luminance properties. The variation in 
neural and vascular responses to identical stimuli is illustrated most clearly in Fig. 6. These data show that the neural retinal and vascular responses differed considerably when the flicker luminance changed from dim-to-bright (Fig. 6A), vs bright-to-dim (Fig. 6B). The vascular counterpart for this neural response profile was a large progressive decrease that totaled $\sim 32 \%$ when flicker luminance changed from dim-to-bright.

The present report that blood flow in the human choroid is linked to light-induced retinal activity is fundamental to our understanding of retinal processing of visual stimuli. This newly defined association between photoreceptor activity and blood flow in the choroid may provide new avenues for research into the pathophysiology of sight-threatening diseases of the eye such as age-related macular degeneration. In addition, this vascular response to photic stimuli may be used for diagnosing hemodynamic abnormalities in the choroid, and also for evaluating the effectiveness of therapeutic procedures aimed at minimizing vision loss.

\section{ACKNOWLEDGMENTS}

This study was funded by grants from the Natural Sciences and Engineering Research Council of Canada \#OGP0116910 (JVL) and \#OGP0121750 (HK), the Fonds de la recherche en santé du Québec (FRSQ) - Vision Network, and the Canadian Optometric Education Trust Fund. HK is the recipient of a Clinician/Scientist award from the FRSQ. All subjects are thanked for their participation in these demanding experiments. The authors also acknowledge the expertise of Normand Lalonde for the machining involved in creating the LED flicker system, the statistical expertise of Francine Giroux, as well as 
Annick Plante, Anne-Michèle Larocque, Marie-Ève Hérard and Claudine Greendale for their help in data collection and analyses. 


\section{REFERENCES}

1. American National Standard for Safe Use of Lasers. ANSI Z136.1 - 2000. $2^{\text {nd }}$ printing 2003. American National Standard. 2003.

2. Ahnelt PK. The photoreceptor mosaic. Eye 12: 531-540, 1998.

3. Bonner RF, and Nossal R. Model for laser Doppler measurement of blood flow in tissue. Appl Optics 20: 2097-2107, 1981.

4. Buerk DG, Riva CE, Cranstoun SD. Frequency and luminance-dependent blood flow and $\mathrm{K}+$ ion changes during flicker stimuli in the cat optic nerve head. Invest Ophthalmol Vis Sci 1995;36:2216-2227.

5. Curcio CA, Sloan KR, Kalina RE, Hendrickson AE. Human photoreceptor topography. J Comp Neurol 1990;292:497-523.

6. Dawson W, Trick GL, and Litzkow C. Improved electrode for electroretinography. Invest Ophthalmol Vis Sci 18: 988-991, 1979.

7. Falsini B, Riva CE, and Logean E. Flicker-evoked changes in human optic nerve blood flow: relationship with retinal neural activity. Invest Ophthalmol Vis Sci 43: 2309-2316, 2002.

8. Fitzgerald ME, Gamlin PDR, Zagvazdin Y, and Reiner A. Central neural circuits for the light-mediated reflexive control of choroidal blood flow in the pigeon eye: A laser Doppler study. Vis Neurosci 13: 655-669, 1996.

9. Flower RW, Fryczkowski AW, and McLeod DS. Variability in choriocapillaris blood flow distribution. Invest Ophthalmol Vis Sci 36: 1247-1258, 1995. 
10. Formaz F, Riva CE, and Geiser M. Diffuse luminance flicker increases retinal vessel diameter in humans. Curr Eye Res 16: 1252-1247, 1997.

11. Fryczkowski AW. Anatomical and functional choroidal lobuli. Int Ophthalmol 18: 131-141, 1994.

12. Fuchsjager-Mayrl G, Polska E, Malec M, and Schmetterer L. Unilateral lightdark transitions affect choroidal blood flow in both eyes. Vision Res 41: 2919-2924, 2001.

13. Garhofer G, Huemer KH, Zawinka C, Schmetterer L, and Dorner GT. Influence of diffuse luminance flicker on choroidal and optic nerve head blood flow. Curr Eye Res 24: 109-113, 2002.

14. Hood D, and Finkelstein MA. Sensitivity to light. In: Handbook of perception and human performance. Vol 1 Sensory processes and perception, edited by Boff KR, Kaufman L, Thomas JP. A Wiley publication; John Wiley and sons. New York, 1986.

15. Kergoat $\mathbf{H}$, and Lovasik JV. The effects of altered retinal vascular perfusion pressure on the white flash scotopic ERG and oscillatory potentials in man. Electroencephalogr Clin Neurophysiol 75: 306-322, 1990.

16. Kiryu J, Asrani S, Shahidi M, Mori M, and Zeimer R. Local response of the primate retinal microcirculation to increased metabolic demand induced by flicker. Invest Ophthalmol Vis Sci 36: 1240-1246, 1995.

17. Linsenmeier RA, and Padnick-Silver L. Metabolic dependence of photoreceptors on the choroid in the normal and detached retina. Invest Ophthalmol Vis Sci 41: 3117-3123, 2000. 
18. Longo A, Geiser M, and Riva CE. Effect of light on choroidal blood flow in the fovea centralis. Klin Monatsbl Augenheilkd 216: 311-312, 2000.

19. Lovasik JV, Kergoat H, Riva CE, Petrig BL, and Geiser M. Choroidal blood flow during exercise-induced changes in the ocular perfusion pressure. Invest Ophthalmol Vis Sci 44: 2126-2132, 2003.

20. Lovasik JV, Kergoat H, and Wajszilber MA. Flicker-induced changes in the choroidal blood flow and electroretinograms. Invest Ophthalmol Vis Sci 44: EAbstract 332. 2003.

21. Marmor MF, and Zrenner E. Standard for clinical electroretinography (1999 update). International Society for Clinical Electrophysiology of Vision. Doc Ophthalmol 97: 143-156, 1998-99.

22. Michelson G, Patzelt A, and Harazny J. Flickering light increases retinal blood flow. Retina 22: 336-343, 2002.

23. Pfeuffer J, van de Moortele PF, Yacoub E, Shmuel A, Adriany G, Andersen P, Merkle H, Garwood M, Ugurbil K, and Hu X. Zoomed functional imaging in the human brain at 7 Tesla with simultaneous high spatial and high temporal resolution. Neuroimage 17: 272-286, 2002.

24. Polak K, Schmetterer L, and Riva CE. Influence of flicker frequency on flickerinduced changes of retinal vessel diameter. Invest Ophthalmol Vis Sci 43: 2721$2726,2002$.

25. Polonsky A, Blake R, Braun J, and Heeger DJ. Neuronal activity in human primary visual cortex correlates with perception during binocular rivalry. Nat Neurosci 3: 1153-1159, 2000. 
26. Riva CE, Cranstoun SD, Grunwald JE, and Petrig BL. Choroidal blood flow in the foveal region of the human ocular fundus. Invest Ophthalmol Vis Sci 35: 4273$4281,1994$.

27. Riva CE, Falsini B, and Logean E. Flicker-evoked responses of human optic nerve head blood flow: luminance versus chromatic modulation. Invest Ophthalmol Vis Sci 42: 756-762, 2001.

28. Riva CE, Titze P, Hero M, Movaffaghy A, and Petrig BL. Choroidal blood flow during isometric exercises. Invest Ophthalmol Vis Sci 38: 2338-2343, 1997.

29. Sasaki Y, Murakami I, Cavanagh P, and Tootell RH. Human brain activity during illusory visual jitter as revealed by functional magnetic resonance imaging. Neuron 35: 1147-1156, 2002. 


\section{FIGURE 1}

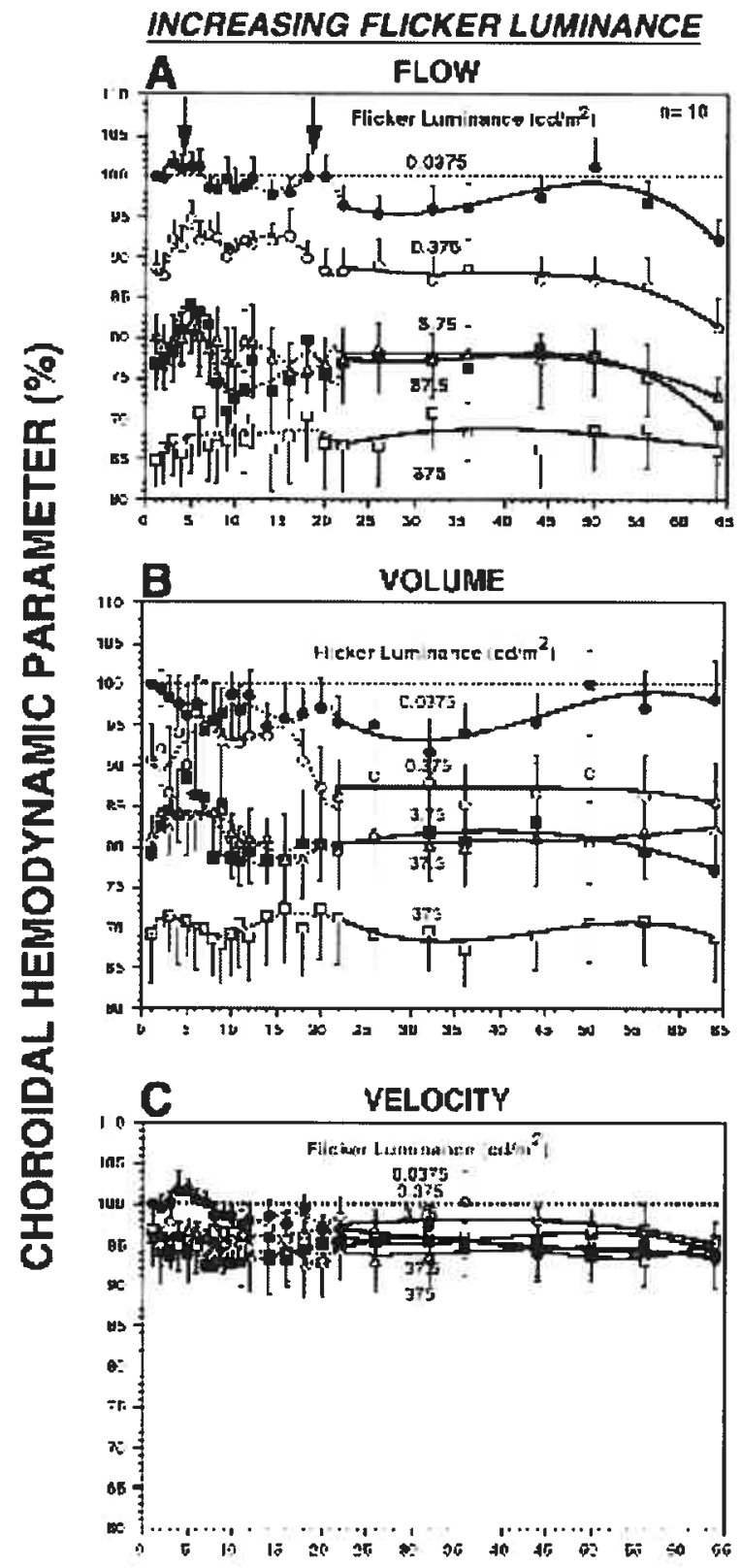

FLICKER FREQUENCY (HZ) 


\section{FIGURE 2}

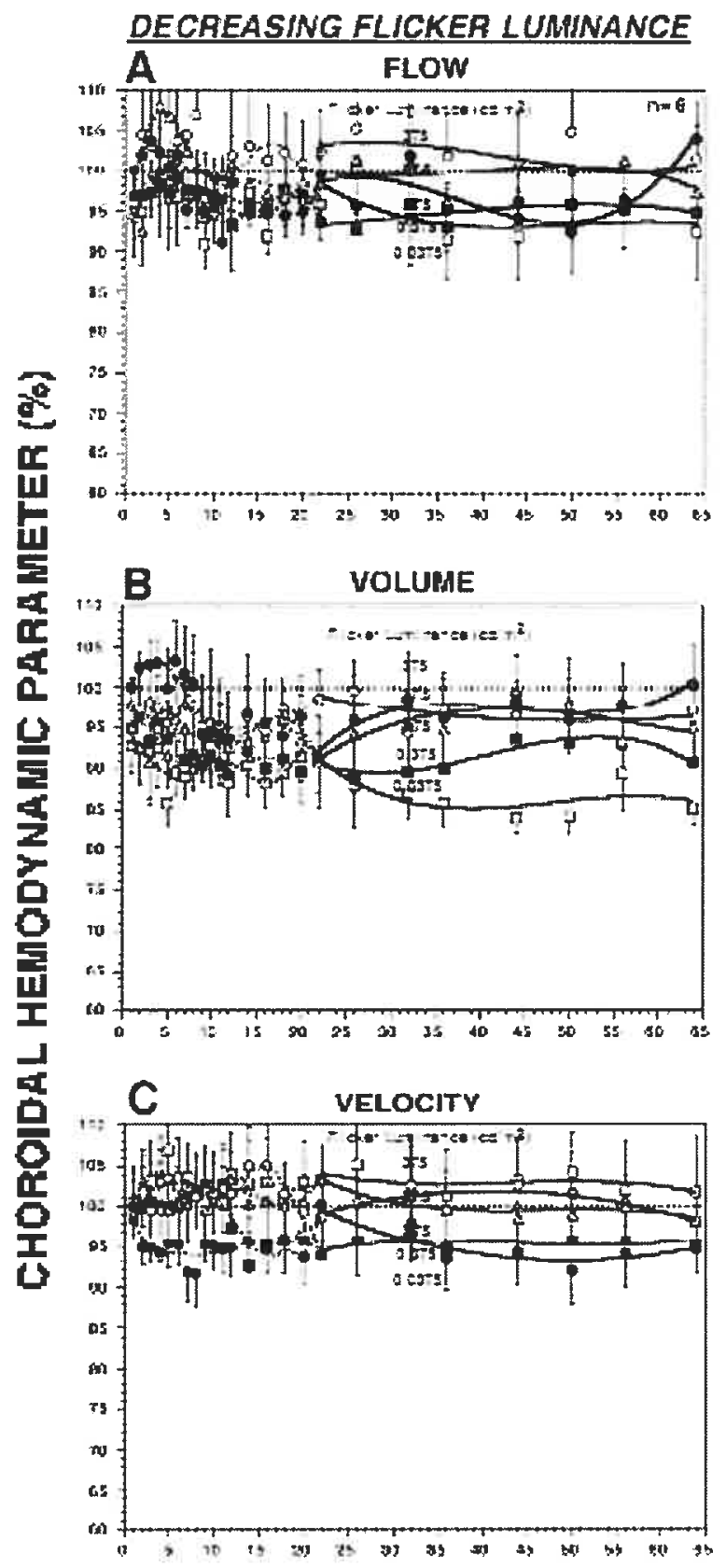

FLICKER FREQUENCY (HZ) 
FIGURE 3

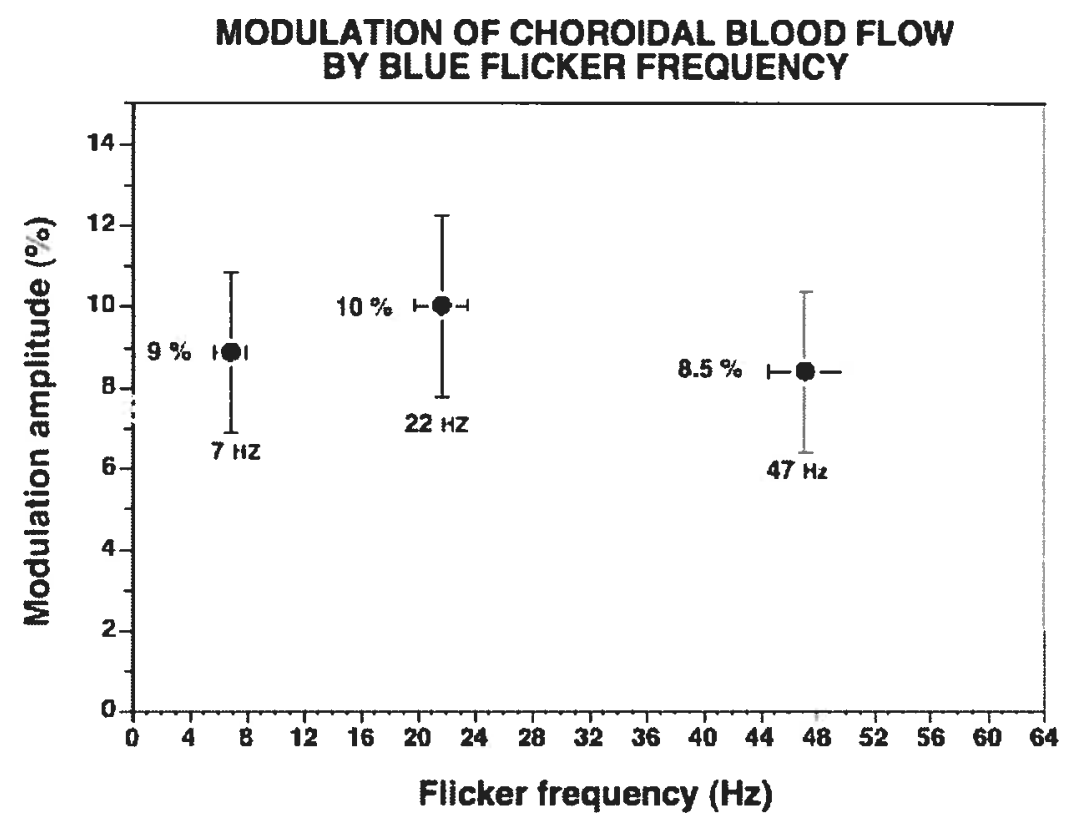




\section{FIGURE 4}

INTERACTIONS BETWEEN THE CHOROIDAL BLOOD FLOW, VOLUME, \& VLOCITY WHEN THE LUHINANCE OF FLICKER FAEQUENCY IS A) INCREASED FROM DIM TO BAIGHT VS B) OECREASEO FROM BRIGHT TO DIM

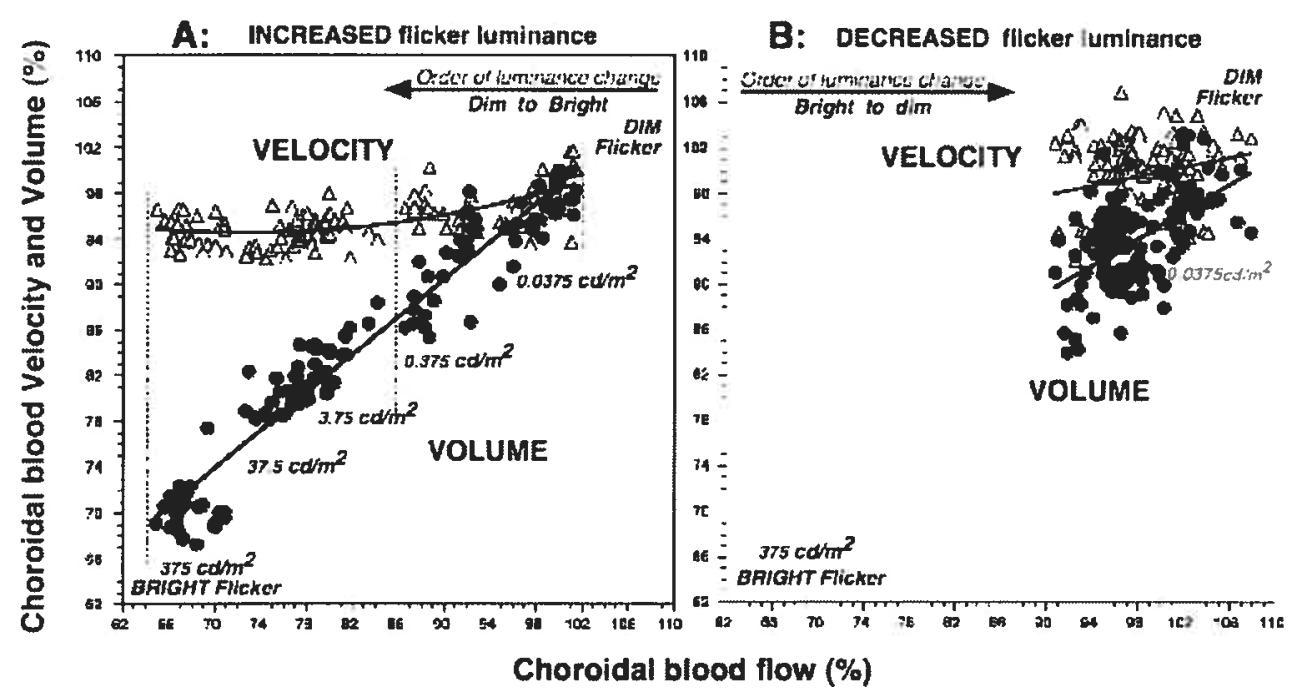




\section{FIGURE 5}

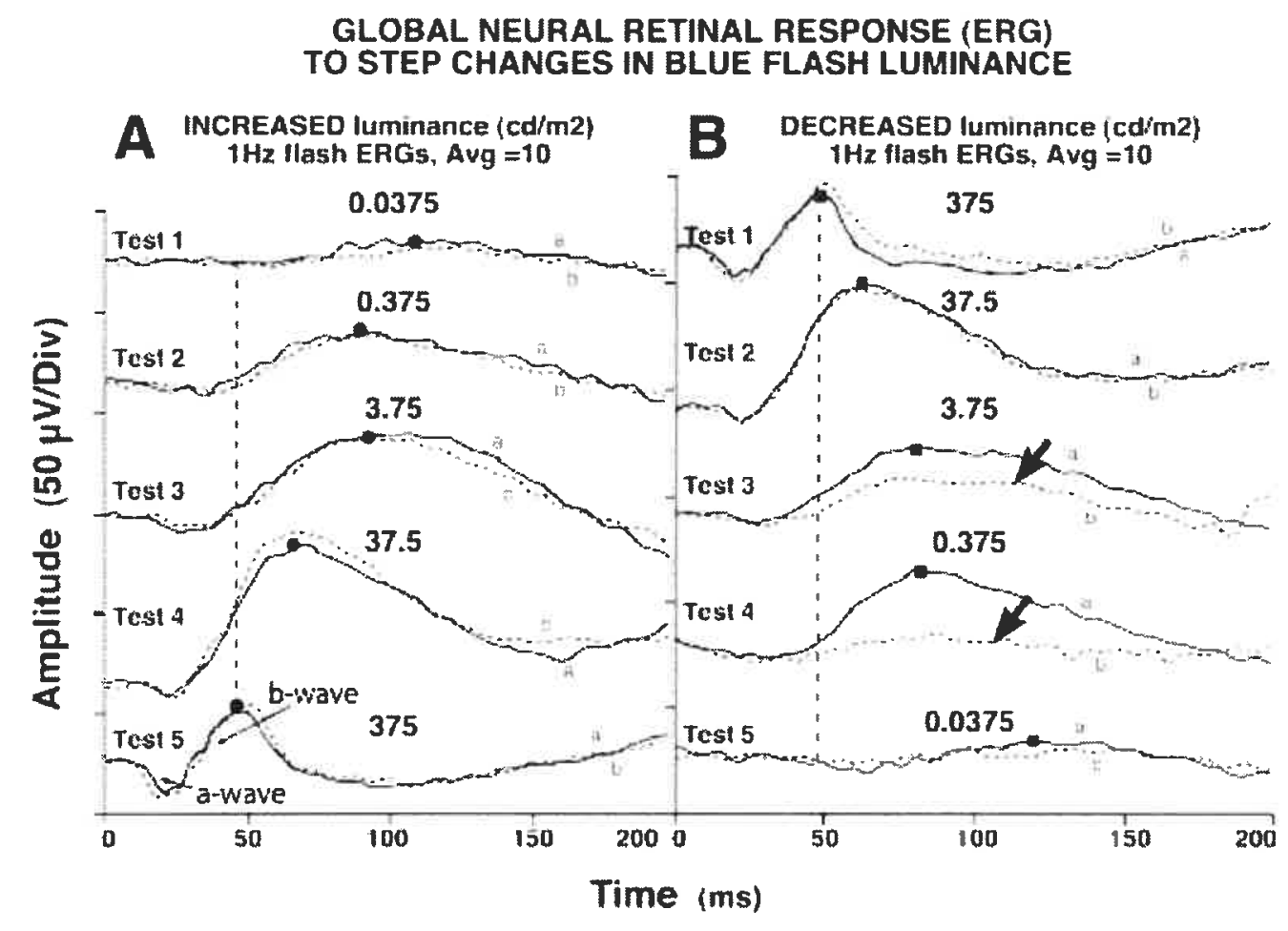




\section{FIGURE 6}

A

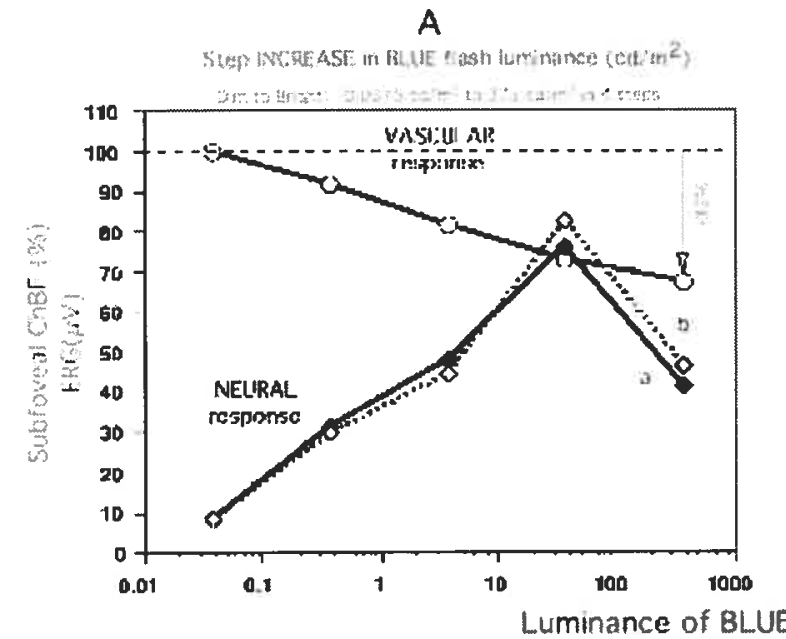

B

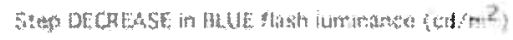

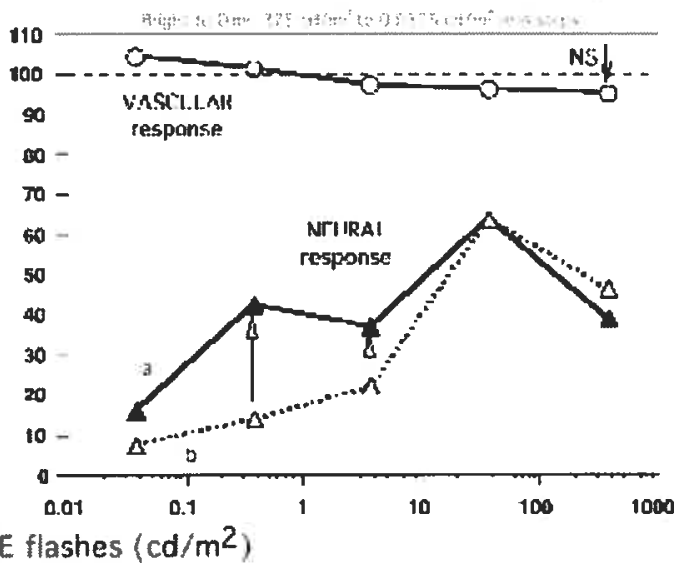




\section{FIGURE CAPTIONS}

Figure 1: Graph illustrating the normalized, group averaged A) choroidal blood Flow, B) Volume and C) Velocity as a function of blue flicker frequency between $1 \mathrm{~Hz}$ and $64 \mathrm{~Hz}$ and $1.0 \log$ unit step increments in luminance. The vertical line through each data point in this figure and all others give the SEM values for that data point. The numbers beside each curve give the flicker luminance value in $\mathrm{cd} / \mathrm{m}^{2}$.

Figure 2: Graph illustrating the normalized, group averaged A) choroidal blood Flow, B) Volume and C) Velocity as a function of blue flicker frequency between $1 \mathrm{~Hz}$ and $64 \mathrm{~Hz}$ and $1.0 \log$ unit step decrements in luminance. The numbers beside each curve give the flicker luminance value in $\mathrm{cd} / \mathrm{m}^{2}$.

Figure 3: Graph illustrating the interaction between blue flicker frequency and ChBF modulation. Significant increments in $\mathrm{ChBF}$ were elicited by 7,22 , and $47 \mathrm{~Hz}$ stimuli.

Figure 4: A) Graph illustrating the interaction between the group-averaged volume and velocity across subjects when the flicker luminance was increased in $1.0 \log$ unit steps from $0.0375 \mathrm{~cd} / \mathrm{m}^{2}$ to $375 \mathrm{~cd} / \mathrm{m}^{2}$. The decrease in ChBF was principally due to a decrease in ChBVol. B) Graph illustrating the interaction between the group-averaged volume and velocity across subjects when the flicker luminance was decreased in 1.0 log unit steps from $375 \mathrm{~cd} / \mathrm{m}^{2}$ to $0.0375 \mathrm{~cd} / \mathrm{m}^{2}$. Neither Volume nor Velocity changed with flicker.

Figure 5: A) Increased luminance: Change in the blue flash ERG waveform, each an average of $10,200 \mathrm{~ms}$ epochs. The small early negative potential and the subsequent large 
positive potential, the "a-wave" and "b-wave" respectively, are identified at the bottom of panel A. The flash luminance, in $\mathrm{cd} / \mathrm{m}^{2}$ is presented atop the corresponding ERG b-wave. The letter " $b$ " defines the ERG status before a blue flicker frequency series. The waveform for the blue flash ERG measured immediately after a blue flicker frequency series is identified by the letter "a".

Figure 5: B) Decreased Luminance: Change in the blue flash ERG waveform, each an average of $10,200 \mathrm{~ms}$ epochs. The flash luminance, in $\mathrm{cd} / \mathrm{m}^{2}$ is presented atop the corresponding ERG b-wave. The letter "b" defines the ERG status before a blue flicker frequency series. The waveform for the blue flash ERG measured immediately after a blue flicker frequency series is identified by the letter " $a$ ".

Figure 6: A) Graphical illustration of the changes in the amplitude of the ERGs shown in Fig. 5 and the simultaneously recorded subfoveal ChBF. As the blue flash increased the ERG amplitude (microvolts) also increased until the flicker luminance was $37.5 \mathrm{~cd} / \mathrm{m}^{2}$ after which the ERG decreased abruptly as it switched from a rod-dominated ERG to a cone-dominated ERG. The simultaneously recorded subfoveal ChBF decreased over the entire range of flicker luminance. B) When the luminance was decreased from high-tolow, the ChBF parameters did not change from baseline. However, the ERG differed in amplitude and waveform before (b) and after (a) each flicker series. The large difference in the retinal response for the 'before' and 'after' flicker ERGs confirmed electrophysiologically that the same blue flash could elicit different retinal responses depending upon retinal light history. Furthermore, the same flicker luminance levels 
elicited very different retinal responses when the order of luminance change was reversed. By extension, these differences in ERGs likely accounted for the different subfoveal $\mathrm{ChBF}$ response profiles elicited by the same flicker intensity but reversed order of luminance steps. 


\section{Conclusion}

The data from the present study revealed that flicker stimulation of the retina from low-to-high luminance in $1.0 \mathrm{log}$ steps over a $5.0 \mathrm{log}$ range was associated with a decrease in subfoveal choroidal blood flow that was proportional to the increase in flicker brightness. As shown in Fig 18, the photic stimulus consisted of a narrow spectral range of blue light that peaked at $473 \mathrm{~nm}$, a region close to the peak spectral response of rhodopsin pigment that is found only in rod photoreceptors.We concluded that the flicker used here exclusively activated the rod population, particularly at low luminance, but likely stimulated blue sensitive cones to some degree at higher luminance flicker levels.

Such a progressive increase in the degree of stimulation of the rod population caused a progressive increase in rod metabolism. Inasmuch as rods populate an annular zone that is concentric to the fovea but some 17 to $20^{\circ}$ distal to the fovea, it was reasonable to assume that this selective activation of rods required an increase in $\mathrm{ChBF}^{(11)}$ in the zone populated by rods so as to meet the increased metabolic demand induced by the flicker. In this regard, with the probing laser being continuously located at the foveola throughout all stimulus conditions, the progressive decrease in the sub-foveal choroidal blood flow with increased flicker luminance is consistent with an autoregulatory process wherein choroidal blood is shunted towards retinal zones with higher metabolic activity and an increased need for metabolites. An examination of blood flow parameters revealed that a decrease in subfoveal choroidal blood flow was caused by a significant decrease in blood volume and a constant flow velocity, indicating a volumetric displacement away from the fovea towards the rods. 
This notion of a physiologically based shunting of blood to areas of the body with increased metabolism has been reported frequently in sports medicine where during stationary bicycling, for example, a disproportionate amount of blood is moved to the large leg muscles to sustain their motor activity. ${ }^{(11)}$ Likewise, in studies of brain physiology using functional MRI measurements of brain activity, it has been reported that different sensory cortical sites "light up" when the corresponding sensory modality has been activated. ${ }^{(26)}$ The jargon of "lighting up" is the equivalent of increased blood flow in a specific area /volume of the brain.

The present study is the first demonstration of an "intelligent" redistribution of choroidal blood in support of increased retinal metabolism at specific retinal zones. The lobular structure of the choriocapillaris would readily allow a volumetric diversion of blood to retinal loci signalling the need for extra metabolites. In the present experiment, eccentrically located photoreceptors were stimulated by using a spectrally narrow beam of blue light that would principally stimulate rod photoreceptors. However, earlier studies ${ }^{(6)}$ examining the effect of light-induced changes in the retinal metabolism on ChBF failed to detect any changes in the subfoveal choroidal blood flow simply because the nature of the stimulus used to activate retinal photoreceptors was inappropriate to show significant changes at the fovea. Specifically, these earlier studies used broadband stimuli such as light from a xenon Grass stroboscope, or a wide spectral band of light to drive retinal neurons. It is therefore not surprising that the subfoveal ChBF did not show significant changes because the stimuli principally activated cone photoreceptors that occur in highest density at the foveal measurement site, and therefore the site requiring increased perfusion to respond to any increase in foveal retinal metabolism. However, Garhofer ${ }^{(6)}$ reported a slight but insignificant increase in subfoveal choroidal blood flow during flicker stimulation of the retina with a $550 \mathrm{~nm}$ low-pass light. Such a stimulus would increase metabolic 
activity in the foveal cones and hence blood flow to that site would increase when the stimulus luminance exceeded the inherent threshold for increased perfusion of the foveomacular zone. Since this study found insignificant changes in the subfoveal choroidal blood flow, the trend of our present findings concerning choroidal blood flow versus flicker luminance suggests that the luminance and flicker frequency was not adequate to elicit changes in $\mathrm{ChBF}$.

It is particularly noteworthy that our results also showed that the same flicker series with step changes in luminance presented in reverse order (bright-to-dim) to the first flicker study (dim-to-bright) failed to elicit a systematic reduction in the choroidal blood flow or volume. These latter results not only confirmed that the reduction in the subfoveal choroidal blood flow were not due to a fatiguing or carry-over effect of repeated stimulation, but that the order of change in flicker luminance caused the retina to respond differently to the same stimulus luminance value. Thus, the light history of a retina has an effect on the subsequent retinal reactivity to photic stimuli.

Proof for the notion that the retina responded differently to the same intensity of the light depending upon its previous light history was provided by electroretinography. The ERG was recorded in response to blue flashes with the same luminance levels used in the blood flow study. The amplitude of the ERG b-wave and it's implicit time were used as indices of the amplitude and speed of the neuro-retinal response. These electrophysiological data confirmed that the amplitude of the retinal response to a stimulus of identical luminance is different when the retina is first exposed to light of increasing luminance versus light of decreasing luminance. Consequently, the degree of $\mathrm{ChBF}$ required to match the metabolic changes induced by light flashes would also vary with the amplitude of the retinal response. These findings provide a clear explanation for the failure of flicker stimuli of decreasing luminance to elicit changes in the subfoveal choroidal blood 
flow beyond $10 \%$ of baseline. As the luminance level increased, the implicit time of the bwave decreased until a dominantly cone response was obtained. The opposite occurred when the luminance was decreased in 1.0 log steps.

Stated otherwise, flicker starting at the highest luminance level predominantly activated centrally located blue as well as other cones, while subsequent decrements in flicker luminance continued to stimulate mainly central cones because the retina had been preexposed to bright light conditions in each preceding step. As a result, these changes in luminance did not elicit a physiological change in the retinal response large enough to require large changes in choroidal blood flow beyond $10 \%$ of baseline that would require shunting of blood towards the periphery.

In conclusion, the focus of the present study was to correlate the changes ins subfoveal $\mathrm{ChBF}$ with changes in retinal metabolism induced by flicker presented across a series of frequencies and luminance levels. These stimulus variables weere used to determine the relative sensitivity of $\mathrm{ChBF}$ to both flicker frequency and flicker luminance.

In human physiology, vascular perfussion and the degree of tissue metabolism are tightly coupled by a complex process of autoregulation of blood supply directly to its functional and metabolic needs.In the brain, functional tasks like somatosensorial stimulation,visual stimulation or even motor tasks induce an increase in cortical blood flow coupled to the increase of local metabolism. ${ }^{(2 ; 26)}$

In recent studies of the intact brain, the regional metabolic activity and blood flow were found to be tightly coupled, but the control mechanism is not fully understood.

\section{Clinical implications:}

The present findings showing that choroidal blood flow has the ability of being redirected to retinal areas with greater metabolic needs and may have significant implications for the 
diagnosis and management of retinal diseases. As an example, patients suffering from diabetic retinopathy evidence a deficit in blood flow (ischemia) to the photoreceptors. Treatment of this condition in some instances implies a laser photodestruction of the peripheral photoreceptors mainly rods to re-direct the the blood flow to preserve the central vision.

In age related macular degeneration (ARMD), local factors such as changes in choroidal blood flow in subfoveal area may lead to hypoxia and consequently induce retinal neovascularization.. We hypothesize that reducing the amount of blue light entering the eye might induce a blood flow to cone populated central areas and delay developpement of ARMD. 


\section{Bibliographie}

1. American National Standard for Safe Use of Lasers. ANSI Z136.1 . 2000.

2. B.Rosengarten SOMK. Overshoot and Undershoot: Control System Analysis of Haemodynamics in a Functional Transcranial Doppler Test. Cerebrovasc Dis . 2002; $14: 148-52$.

3. Brigell M, Bach M Barber C Moskowitz A Robson J. Guidelines for calibration of stimulus and recording parameters used in clinical electrophysiology of vision. Doc Ophthalmol 107, 185-193 . 2003.

4. Bron, A. J. and Tripathi, R. Wolff's Anatomy of the Eye and Orbit. 97. Arnold.

5. Cornsweet T N. Visual Perceptions. 2 ed. New York: New York Academic Press, 1971:

6. Garhofer G, Huemer K Zawinka C Schmetterer L Dorner G. Influence of diffuse luminance flicker on choroidal and optic nerve head blood flow. Current Eye Research 24, 109-113. 2002.

7. Garhofer G, Zawinka Resch H Huemer K Dorner G Schmetterer L. Diffuse luminance flicker increases blood flow in major retinal arteries and veins. Vision Research 44, 833-838. 2004.

8. Geiser M, Moret F Riva Ch. Helmet mounted choroidal laser Doppler flowmeter. SPIE 4263, 91-97. 2001.

9. Hayreh S.S. Posterior Ciliary Artery Circulation in Health and Disease-The Weisenfeld Lecture. IOVS. 2004;45:749-57.

10. Hogan MJ, Alvarado JA Weddell JE. Histology of the human eye. Philadelphia: W.B. Saunders, 202-255. 71. 
11. Lovasik J., Kergoat H., Riva C., Petrig B. and Geiser M.,. Choroidal Blood Flow during Exercise-Induced Changes in the Ocular Perfusion Pressure. Invest. Ophthalmol. Vis. Sci 44, 2126-2132.

12. Kaufman P., Alm A. Adler's Physiology of the Eye. Ed. Mosby. 10th edition.ISBN 0323011365

13. Kiel J. Choroidal Myogenic Autoregulation and Intraocular Pressure. Exp Eye Res. 58, 529-544. 94.

14. Kiel J W, Van Heuven W A J. Ocular Perfusion Pressure and Choroidal Blood Flow in the Rabbit. IOVS 36, 579-585. 95.

15. Linsenmeier R, Padnick-Silver L. Metabolic dependence of photoreceptors on the choroid in the normal and detached retina. IOVS 41(10), 3117-3123. 2000.

16. Longo A, Geiser M Riva Ch. Subfoveal choroidal blood flow in response to lightdark exposure. IOVS 41(9), 2678-2683. 2000.

17. Marmor MF, Holder GE Seeliger MW Yamamoto S. Standard for clinical electroretinography (2004 update). Documenta Ophthalmologica 108, 107114. 2004.

18. Peter Sterling. Deciphering the Retinal's wiring diagram. Nature America 2 (10),

851-3. 99 .

19. Petrig B, Riva Ch. Near infrared retinal laser Doppler velocimetry and flowmetry. Applied Optics 30, 2073-2078. 91. 
20. Petrig B, Riva Ch. Laser Doppler flowmetry in the optic nerve head.Principle and technique . PillunatL,Harris A,Anderson D, Current concepts on ocular blood flow in glaucoma.The Hague,Netherlands ,Kugler publications (10), 15541562. 99.

21. Polak K, Schmettere L Riva Ch. Influence of flicker frequency on Flicker induced changes of retinal vessel diameter. IOVS 43(8), 2721-2726. 2002.

22. Polak K, Wmpissinnger B Berisha F Georgopoulos M Schmetterer L. Effects of sildenafil on retinal blood flow and flicker - induced retinal vasodilation in healthy subjects. IOVS 44(11), 4872-4876. 2003.

23. Riva C, Ross B Benedek G. Laser Doppler measurements of blood flow in capillary tubes and retinal arteries. IOVS 11(11), 936-944. 72.

24. Riva Ch. Basic principles of laser Doppler flowmetry and application to the ocular circulation. International Ophthalmology 23, 183-189. 2001.

25. Riva Ch, Hero M Titze P Petrig B. Autoregulation of human optic nerve head blood flow in response to acute changes in ocular perfusion pressure. Graefe's Arch Clin Ophthalmol 235, 618-626. 97.

26. Rosengarten $\mathrm{B}$, Lutz $\mathrm{H}$ b Hossmann $\mathrm{K}$. A control system approach for evaluating somatosensory activation by laser-Doppler flowmetry in the rat cortex. Journal of Neuroscience Methods 130, 75-81. 2003. 
27. Roy C, Sherrington C. On the regulation of the blood supply of the brain. J Physiology 11(85-108). 1890.

28. Schmetterer L ,Dallinger S,Findl O.,Strenn K.,Graselli U., Eischler H.,Woltz M. Noninvasive investigations of the normal ocular circulation in humans. IOVS. 1998;39:1210-20.

29.Shimizu K. Structure of ocular vessels. New York: Igaku-Shoin, 1978:

30.Stiris T, Christensen T Bratlid D. Effect of different phototherapy lights on retinal and choroidal blood flow.Dev Pharmacol Ther 17(1991), 70-78. 91.

31.Wise G., Dollery C. Henkind P. The retinal circulation. 85-117. 71. 1971, Harper \& Row.

32. Parver L,Auker C.,Carpenter D.,Choroidal blood flow as a heat dispatching mechanism in the choroid AJO (1980) 89,641-646 


\section{PRESENTATION OF THE FLICKER STIMULATOR ELECTRONIC CIRCUIT}

\section{The Flicker controller}

The flicker stimulator was designed to provide a square wave luminous output in order to drive an array of 5 high intensity light emitting diodes.

Various important features were incorporated into the circuit design:

-Stability over time and temperature

-Very short rise time and fall time

-Generation of a square wave light pulse.

-Versatility and upgradeability

The circuit was designed around the 555 timer integrated circuit that served as a clock generator to run freely in astable condition.

This chip has the capability of sensing the "state of charge "of capacitor C .

Figure A: Typical astable oscillator configuration

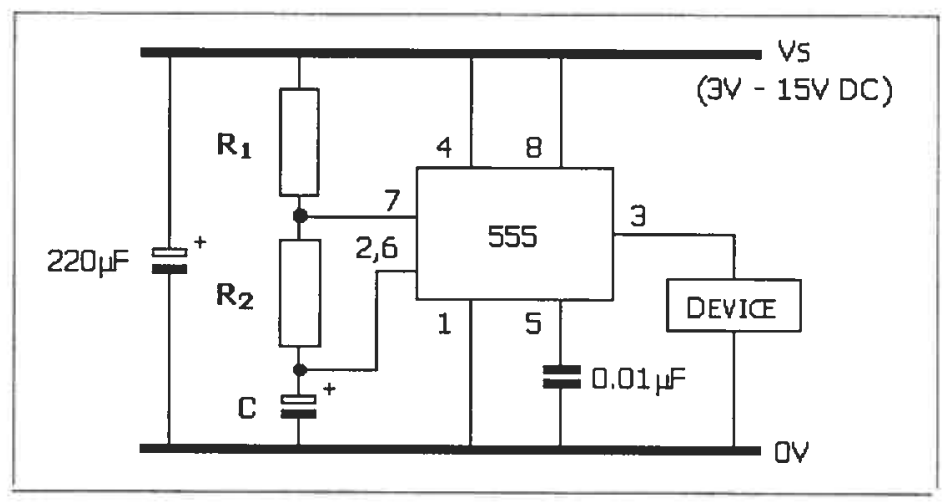


When the capacitor is charged to its $2 / 3$ of total capacity, threshold is reached and the internal flip flop is triggered to a high position output.

This charge is regulated by a resistor (R1) such that a change in resistance to specific values will result in different charging rates or time constants. The capacitor $\mathrm{C}$ is discharged trough R2 until $1 / 3$ of the voltage is reached, and then the cycle restarts.

The frequency constant was calculated upon the formula :

$$
\text { Frequency } f=\frac{1.44}{(\mathrm{R} 1+2 \times \mathrm{R} 2)}
$$

Figure \# B: LM555 internal schematic diagram

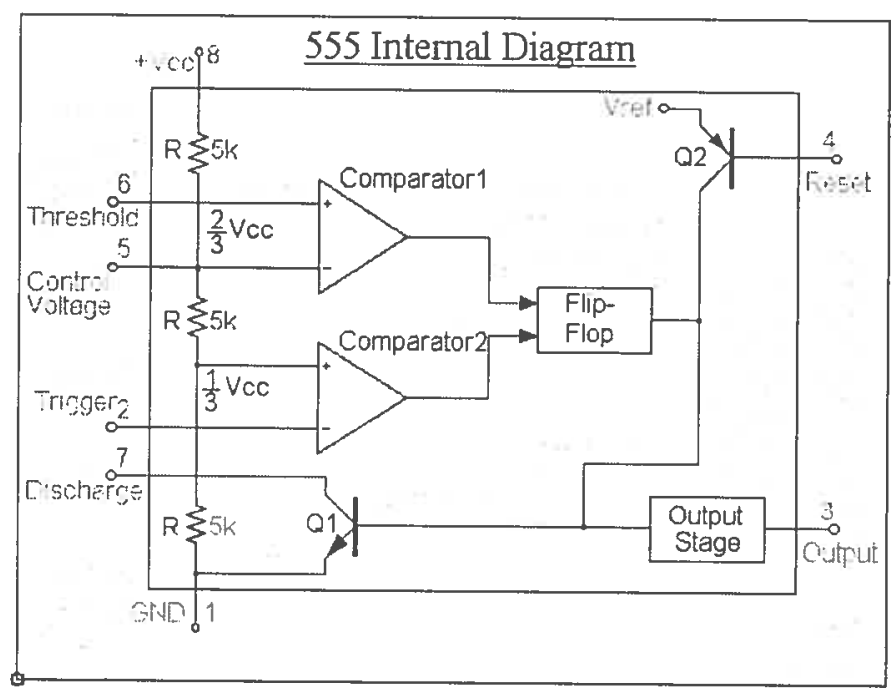

The time constants and frequencies were calibrated with oscilloscope measurements (BK Precision, USA) as well as with a Fluke 1900A multi-counter frequency meter (Fluke,USA.). 
In the present flicker design, two clock generators were used as shown in the operational diagram, the first one for low frequencies from 1 to $12 \mathrm{Hertz}$ and the second clock is set to frequencies from 14 to 64 Hertz.

The duty cycle (ON/OFF) output is calculated on the basis of the formula:

$$
\begin{aligned}
\text { HIGH TIME } & =0.69(\mathrm{R} 1+\mathrm{R} 2) \mathrm{C} \\
\text { LOW TIME } & =0.69(\mathrm{R} 2 \times \mathrm{C})
\end{aligned}
$$

Therefore, if $\mathrm{R} 1$ is kept at $1 \mathrm{Kohm}$ we can easily obtain a duty cycle of $50 \%$

$$
\text { DUTY CYCLE }=\frac{\text { HIGH TIME }}{\text { PULSE PERIOD }}
$$

Fig \# C Block diagram of flicker system

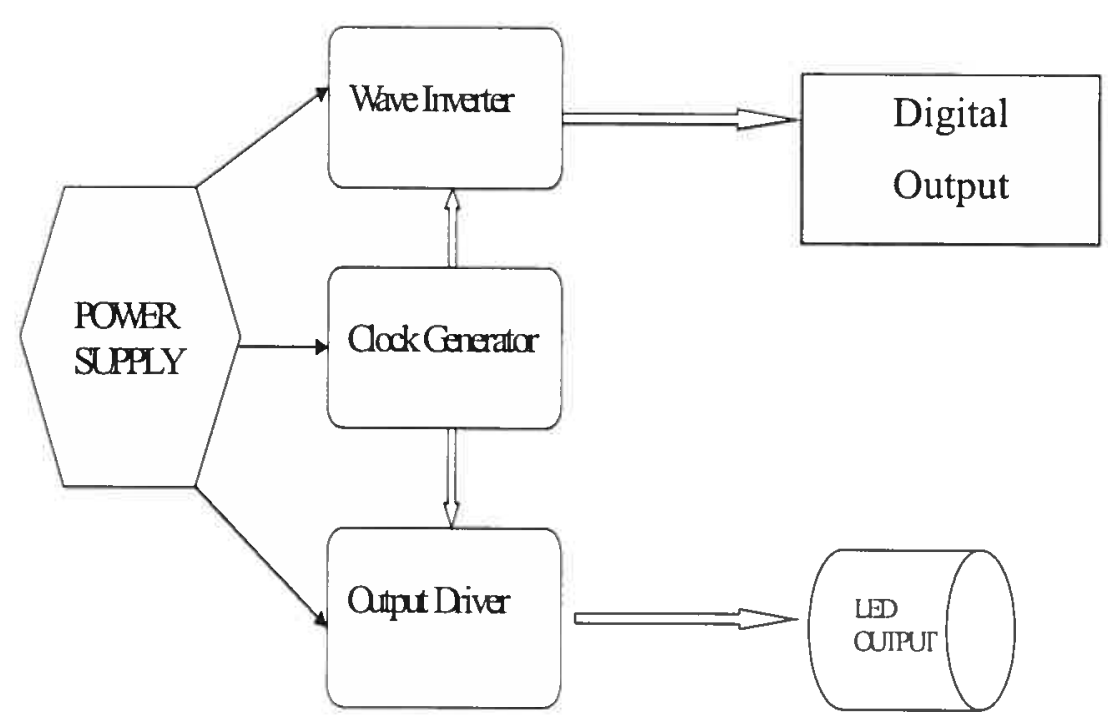


Special care was taken in the circuit design to assure a modulation depth of $100 \%$ to deliver a full ON-OFF stimulus.

As the current drained for the LED array is $150 \mathrm{~mA}$ and due to the high repetition rate a fast switching Mosfet transistor was used to sequentially select the output of one of the two oscillating clocks to power up the flickering LED's. Furthermore, this output is used to discharge a Schmitt trigger inverter in order to get a mirrored square wave TTL signal to synchronize with the Espion electroretinogram (ERG) system.

The power supply of the unit has the advantage of a single chip voltage stabilizer, giving a constant output of 5 volts to power both clocks, power driver transistor and LEDs separately.

\section{Operation}

The circuit board and power supply is self contained in a grounded metal box and all wires to the LED array were shielded to prevent from spurious induction noise.

Four LED's arrays were mounted in a carrousel in a metal box attached to the fundus camera in order to provide 4 different light colors (blue, red, green, white) as seen in figure C.The LED emitted light was re-directed by mean of a beamsplitter into the illumination pathway of the fundus camera and projected into the eye in Maxwellian view. 
The circuit was manually controlled by the operator who is able to select the desired flicker frequencies by turning switch $\mathrm{SW}_{1}$ or $\mathrm{SW}_{2}$ sequentially according to the desired frequency. The frequency sequence is as follows:

\begin{tabular}{|l|l|l|l|l|l|l|l|l|l|l|l|l|l|l|l|l|l|l|l|l|l|l|l|l|}
\hline $\begin{array}{l}\text { Switch } \\
\text { position }\end{array}$ & 1 & 2 & 3 & 4 & 5 & 6 & 7 & 8 & 9 & 1 & 1 & 1 & 1 & 1 & 1 & 1 & 1 & 1 & 1 & 2 & 2 & 2 & 2 & 2 \\
\hline $\begin{array}{l}\text { Frequency } \\
\text { in } \mathrm{Hz}\end{array}$ & 1 & 2 & 3 & 4 & 5 & 6 & 7 & 8 & 9 & 1 & 1 & 1 & 1 & 1 & 1 & 2 & 2 & 2 & 3 & 3 & 4 & 5 & 5 & 6 \\
\hline
\end{tabular}

Switch $\mathrm{SW}_{3}$ provides an override of the flicker to get a continuous full intensity light for calibration and focusing purposes.

\section{The Flicker Stimulus}

The flicker stimulator is composed of four LED's light sources mounted on a carrousel with a rotating knob as shown in Fig D. Each light source has 5 high intensity LED's symmetrically distributed and a specific color wavelength. Therefore it has the capability of changing flicker colors to blue, red, green, or white.

This carrousel is mounted in a shielded metal box to protect from any electrical noise interference. By means of a split prism the output beam is re directed into the illumination pathway of the fundus camera . 
Figure \#D Diagram of coupling the probing laser beam and the flicker stimulation

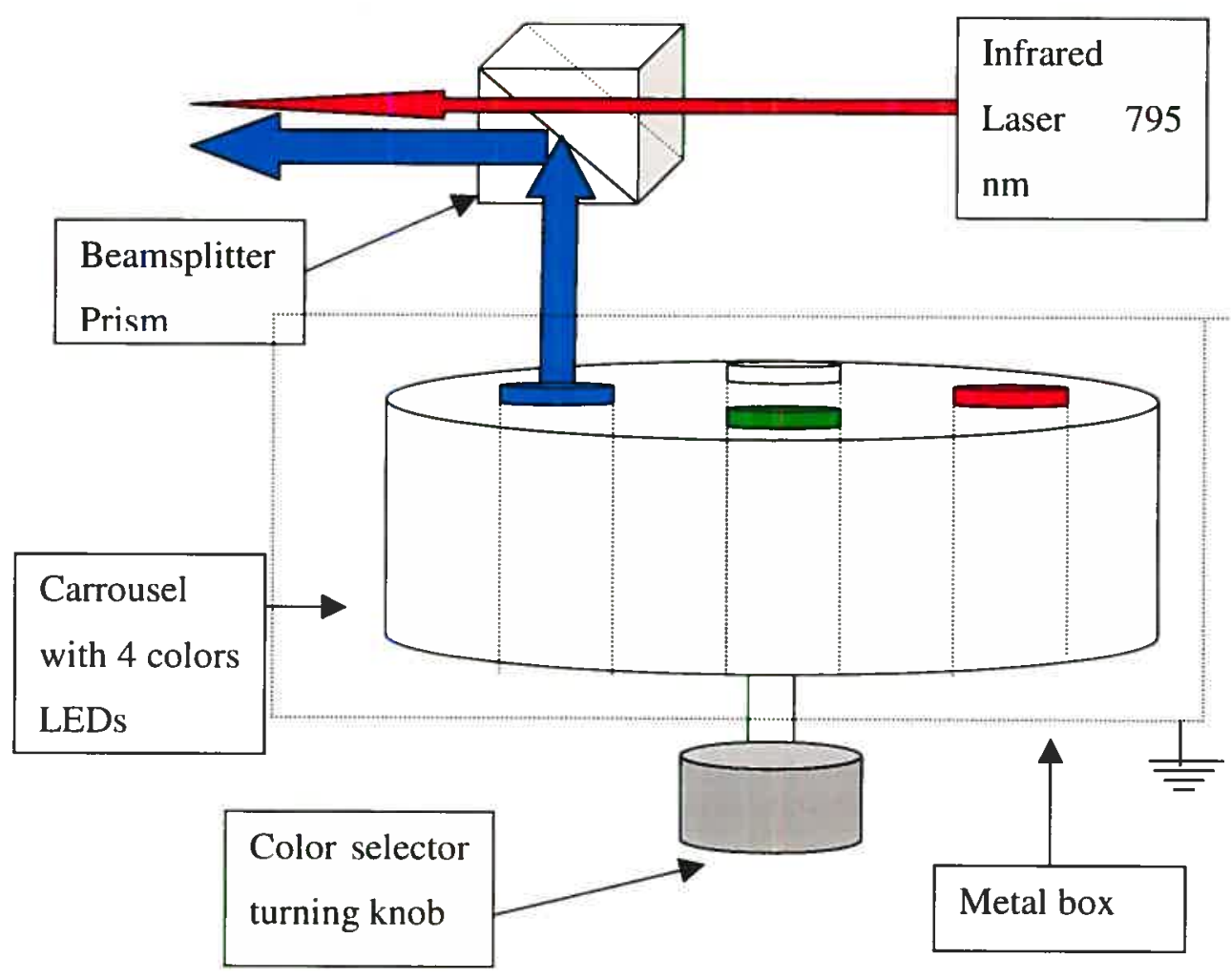

Once the system was mounted and calibrated on the fundus camera, the maximum output measured at cornea level was $375 \mathrm{~cd} / \mathrm{m} 2$ as detected with Minolta light meter -10 (Minolta ,Japan).

To perform our study and to obtain different and reliable luminance levels we introduced neutral density Wratten filters (Kodak,USA) in the illumination pathway. Densities were 4-3-2 and $1 \log$ units. The pulse duration as a function of frequency is graphed below. 


\section{Pulse duration vs frequency}

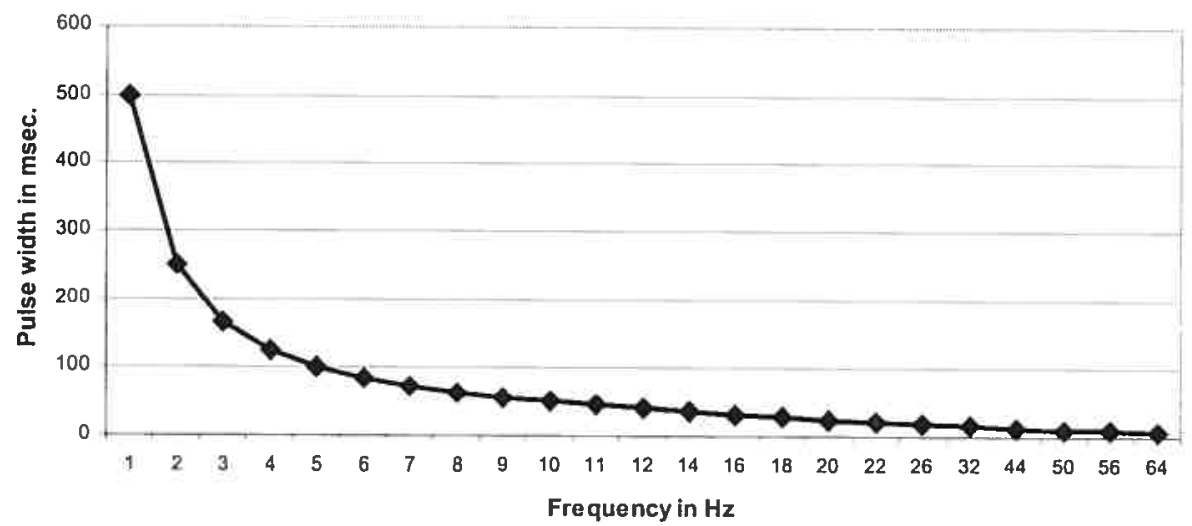

Figure \# E Complete circuit diagram for flicker control
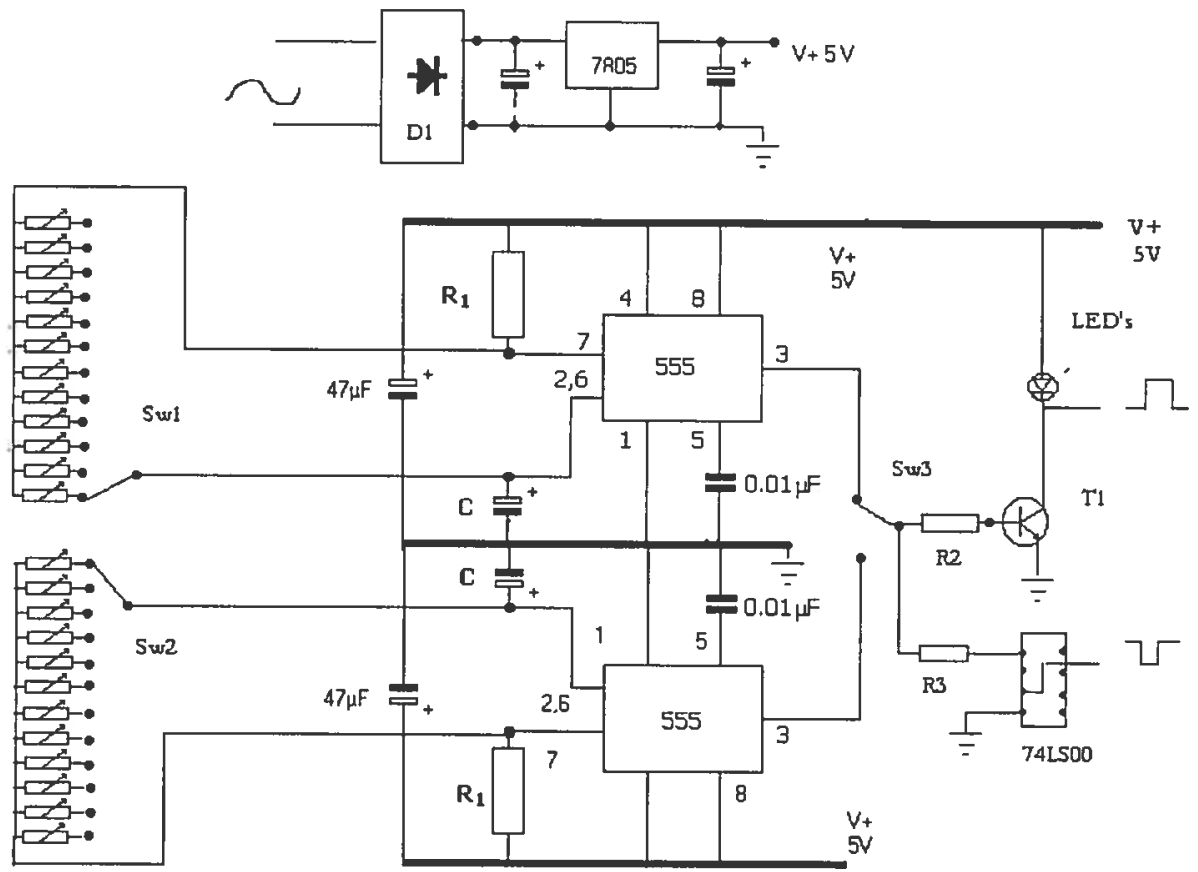